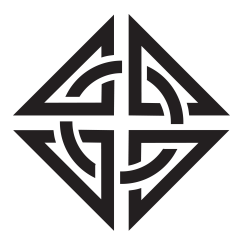

SCIENTIA
Sharif University of Technology

Scientia Iranica

Transactions E: Industrial Engineering

http://scientiairanica.sharif.edu

\title{
Multi-criteria group decision-making method based on generalized power harmonic aggregation operators with normal intuitionistic fuzzy numbers
}

\author{
H.-G. Peng ${ }^{\mathrm{a}}$, J. Wang ${ }^{\mathrm{b}, *}$, and J.-Q. Wang ${ }^{\mathrm{a}}$ \\ a. School of Business, Central South University, Changsha 410083, PR China. \\ b. College of Logistics and Transportation, Central South University of Forestry and Technology, Changsha 410004, China \\ Received 28 September 2018; received in revised form 6 October 2019; accepted 23 December 2019
}

\author{
KEYWORDS \\ Normal intuitionistic \\ fuzzy numbers; \\ Multi-criteria group \\ decision-making; \\ Power average \\ operator; \\ Harmonic mean \\ operator; \\ Generalized \\ aggregation operator.
}

\begin{abstract}
Normal Intuitionistic Fuzzy Number (NIFN), which is introduced based on intuitionistic fuzzy sets and normal fuzzy numbers, is a useful tool for presenting uncertain information under complicated situations. This study focuses on the development of an effective method by combining NIFNs with the power average and harmonic mean operators to address Multi-Criteria Group Decision-Making (MCGDM) problems, wherein weight information is completely unknown. First, an effective ranking method for NIFNs is provided in view of defects of the existing comparison method of NIFNs. Subsequently, three normal intuitionistic generalized power harmonic aggregation operators are proposed based on the operations of NIFNs. Next, a new MCGDM method is developed. Finally, a numerical example concerning coal mine safety evaluation is provided for demonstration. The feasibility and validity of the proposed method are further verified by sensitivity analysis and comparison with other existing methods.
\end{abstract}

(C) 2021 Sharif University of Technology. All rights reserved.

\section{Introduction}

Multi-Criteria Decision-Making (MCDM) refers to making a decision based on many irreplaceable criteria under a complicated and uncertain environment. The purpose of MCDM is to identify the best alternative from a finite set of distinct alternatives. Decisionmaking problems have become increasingly complex with the development of society. Individual decisionmaking has become inefficient as the uncertainty of

*. Corresponding author. Tel.: +86 73185623292 E-mail addresses: hg_peng@csu.edu.cn (H.-G. Peng); wangjing@csuft.edu.cn (J.Wang); jqwang@csu.edu.cn (J.-Q. Wang)

doi: $10.24200 /$ sci.2019.51897.2423 these problems increased. To provide a feasible solution to complicated decision-making problems, the opinions of Decision-Makers (DMs) or experts from various fields must be unified. In this case, Multi-Criteria Group Decision-Making (MCGDM) method has been developed and applied widely $[1,2]$.

To describe the fuzziness of decision information, Zadeh [3] proposed Fuzzy Set (FS), which is considered a useful tool for solving practical problems $[4,5]$. Although FSs are capable of dealing with fuzzy information, there is a limitation that FSs describe information by membership degree only. However, in some cases, membership degree alone cannot precisely describe information in practical problems. To address this issue, Atanassov [6] introduced Intuitionistic Fuzzy Sets (IFSs), which add a non-membership degree to character fuzzy information. IFS is an effective infor- 
mation description tool and has been applied in various fields $[7-10]$.

It is worth noting that the information described by FSs and IFSs is limited in the discrete domain. To extend these sets to the continuous domain, Shu et al. [11] introduced the concept of Triangular Intuitionistic Fuzzy Numbers (TIFNs). Subsequently, the operations and ranking method of TIFNs were studied $[12,13]$, and an extended VIKOR method for MCGDM problems with TIFNs was developed [14]. Moreover, to extend the TIFNs, Wang and Zhong [15] proposed the concept of Trapezoidal Intuitionistic Fuzzy Numbers (TrIFNs). TrIFNs were then studied by many scholars $[16,17]$ and applied to address MCGDM problems [18].

In reality, a large number of natural and social phenomena comply with normal distribution. In this case, Normal Fuzzy Numbers (NFNs) [19] were introduced to appropriately present such phenomena. Compared with triangular fuzzy numbers and trapezoidal fuzzy numbers, NFNs have much more power in practical application [20]. In view of this, Wang et al. [21] introduced Normal Intuitionistic Fuzzy Numbers (NIFNs) by integrating NFNs with IFS. NFNs have many advantages over other fuzzy numbers, and NIFNs are also superior to other intuitionistic fuzzy numbers [22]. In NIFNs, membership and non-membership functions depend on NFNs, and the membership and non-membership degrees are no longer merely related to a fuzzy concept of "good" or "bad". On the basis of NIFNs, Wang et al. [21] defined the score function, operations, and similarity measure and a series of normal intuitionistic aggregation operators. Liu and Teng [23] further proposed Normal IntervalValued Intuitionistic Fuzzy Numbers (NIVIFNs) and developed many generalized aggregation operators.

Aggregation operators are important tools for information fusion in decision-making problems, and many aggregation operators, such as the weighted average operator [24,25], weighted geometric average operator [26,27], Ordered Weighted Average (OWA) operator [28,29], prioritized average operator [30,31], hybrid aggregation operator [32,33], Bonferroni mean operator [34], and generalized aggregation operator [35-37] were studied and applied widely. In terms of IFSs, Xu [38] introduced the intuitionistic fuzzy weighted averaging operator. $\mathrm{Xu}$ and Yager [39] proposed the intuitionistic fuzzy weighted geometric operator. Zhao et al. [40] presented the generalized intuitionistic fuzzy weighted averaging operator. In the aspect of TIFNs, Zhang and Liu [41] proposed the triangular intuitionistic fuzzy weighted arithmetic averaging operator; Wang et al. [42] developed the logic AND and OR operators for TIFNs by combining the OWA and probability operators; and $\mathrm{Yu}$ [43] defined the triangular intuitionistic fuzzy prioritized weighted average operator. In terms of TrIFNs,
Wang and Zhong [15] defined the trapezoidal intuitionistic fuzzy weighted arithmetic averaging operator. Wei [44] proposed the trapezoidal intuitionistic fuzzy ordered weighted averaging operator. Wu and Cao [45] presented the trapezoidal intuitionistic fuzzy ordered weighted geometric operator. In terms of NIFNs, Wang et al. [21] defined the Normal Intuitionistic Fuzzy Ordered Weighted Averaging (NIFOWA) operator and the Induced Normal Intuitionistic Fuzzy Related Ordered Weighted Averaging (INIFROWA) operator. Wang et al. [22] proposed the Normal Intuitionistic Fuzzy-Induced Generalized Ordered Weighted Averaging (NIFIGOWA) operator. Liu and Teng [23] further developed the normal interval-valued intuitionistic fuzzy weighted arithmetic averaging operator.

However, none of the aforementioned fuzzy aggregation operators consider the interrelationships between variables and the central tendency data in the aggregation process. Introduced by Yager [46], the Power Average (PA) operator accounts for information regarding the interrelationships and input variables and allows these variables to support each other in the aggregation process. In this way, $\mathrm{Xu}$ and Yager [47] proposed new geometric aggregation operators including the power geometric operator and the power ordered weighted geometric operator. Subsequently, Xu [48] proposed the intuitionistic fuzzy power weighted average operator. $\mathrm{Li}$ et al. [49] defined the linguistic neutrosophic power weighted aggregation operators. Moreover, Harmonic Mean (HM) is a conservative average operator, which is a useful tool for aggregating central tendency data and can be used to provide aggregation lying between the maximum and minimum operators [50]. Motivated by this idea, Liu et al. [51] introduced the ordered weighted harmonic averaging operator. Wei [52] proposed the triangular intuitionistic fuzzy ordered weighted HM operator. Liu et al. [53] developed the generalized interval-valued trapezoidal fuzzy weighted harmonic averaging operator.

NIFNs enjoy a significant capability in describing decision-making information and can precisely reflect the uncertainty and fuzziness of information of different dimensions. For example, an expert is asked to estimate human being's lifetime in the next ten years. The expert states that the human being's lifetime obeys normal distribution $N=(80,20)$. Moreover, the expert has $60 \%$ confidence that the estimation is correct, $20 \%$ confidence that the estimation is wrong, and $20 \%$ indeterminacy about this estimation. In this case, the expert's evaluation can be depicted as a NIFN $\langle(80,29), 0.6,0.2\rangle$. Although some normal intuitionistic fuzzy aggregation operators have been proposed, they cannot handle the relationships among data in the aggregation process. In fact, aggregated values are correlated in many practical problems and the central tendency data plays a vital role in decision results [54]. 
For example, in coal mine safety evaluation problems, environmental security may be affected by geological condition, and human diathesis may be affected by management level; then, the evaluation values under these criteria are correlative. Moreover, for a specific alternative, suppose that ten evaluation values of 6,5 , $6,2,7,5,10,6,7$, and 6 are provided for it under ten criteria. Compared with 5,6 , and 7 , evaluation values 2 and 10 are improperly low and high. In this case, 5, 6 , and 7 can be regarded as the central tendency data, and 2 and 10 are exceptional data. To deal with these problems, interrelationships among variables should be considered and the importance of central tendency data must be highlighted. Therefore, the primary objective of this study is to combine PA with HM under normal intuitionistic fuzzy environments to develop a series of useful aggregation operators to address practical problems effectively.

Based on the analysis above, the primary motivations of this study are summarized as follows:

1. Compared with TIFNs and TrIFNs, NIFNs possess the following advantages. First, NIFNs are highly applicable to present practical decision information because normal distribution can explain many social phenomena and human activities. Second, NIFNs have favorable mathematical properties because the higher derivative of the normal membership function is continuous. Third, the fuzzy concepts described by the normal membership and non-membership functions are closer to human mind [20]. In conclusion, NIFN is a significantly useful tool for presenting assessments under uncertain or fuzzy environment. Therefore, this study introduces NIFN as a mean to depict decision information involved in practical problems. Moreover, in view of the defects of the existing comparison method of NIFNs, which will be discussed in Section 3 in detail, this study develops a valid ranking method to improve the accuracy of decision results;

2. In practical MCGDM problems, the input variables may be correlative and some excessively low or improperly high evaluation values may be provided by DMs because of their subjective prejudice. Previous discussion indicates that PA enables the correlative variables to support each other, and $\mathrm{HM}$ can relive the impact of exceptional data in the aggregation process; the two operators are highly useful tools for information fusion. However, there is a lack of aggregation operator that can simultaneously consider the interrelationships among variables and central tendency data. Moreover, the existing normal intuitionistic fuzzy aggregation operators cannot handle the relationships among data in the aggregation process. To develop effective and applicable methods for solving practical problems, this study focuses on integrating PA with HM under normal intuitionistic fuzzy environments to propose a series of useful aggregation operators.

The rest of this study is organized as follows. In Section 2, some basic concepts are reviewed briefly and the distance and operations of NIFNs are introduced. In Section 3, an effective ranking method for NIFNs is presented. In Section 4, three normal intuitionistic generalized aggregation operators are developed. In Section 5, a new MCGDM method under normal intuitionistic fuzzy environment is developed. In Section 6, an illustrative example is used to verify the proposed method. Sensitivity analysis and comparison analysis are then conducted. Finally, the conclusion is presented in Section 7.

\section{Preliminaries}

In this section, some basic concepts including IFS, NFNs, NIFNs, and the corresponding distance and operations are introduced.

\subsection{Intuitionistic Fuzzy Set (IFS)}

IFS is a contributing concept to the field of fuzzy decision-making and is of utmost significance to the introduction of NIFNs. The definition of IFS is presented in the following:

Definition 1 [6]. Let $X$ be a universe of discourse and then, an IFS $A$ in $X$ is defined as:

$$
A=\left\{<x, u_{A}(x), v_{A}(x)>\mid x \in X\right\}
$$

where $u_{A}: x \rightarrow[0,1]$ represents the membership degree of $x$ to $A, v_{A}: x \rightarrow[0,1]$ represents the nonmembership degree of $x$ to $A$, and $0 \leq u_{A}(x)+v_{A}(x) \leq$ 1. In general, $\pi_{A}(x)=1-u_{A}(x)-v_{A}(x)$ can be called the hesitation degree of $x$ to $A$. When $\pi_{A}(x)=0$ for each $x \in X$, the IFS is reduced to a FS.

\subsection{Normal Intuitionistic Fuzzy Number $(N I F N)$}

In reality, normal distribution can illustrate many natural and social phenomena, and it presents universal applicability and significant influence in various fields, such as mathematics, economics, and engineering. In view of this, normal distribution is used to characterize fuzzy information, and the concepts of NFN and NIFN are proposed.

Definition 2 [21]. Let $X$ be a non-empty real number set and $A=(a, \sigma)$ be an NFN if its membership function satisfies:

$$
A(x)=e^{-\left(\frac{x-a}{\sigma}\right)^{2}},(\sigma>0),
$$

and the set of NFNs can be considered as $\tilde{N}$. 
$A(x)$ is similar to the probability density function $f(x)=\frac{1}{\sqrt{2 \pi} \theta} e^{-\frac{(x-\alpha)^{2}}{2 \theta^{2}}}$ of normal distribution, $a$ and $\sigma$ in $A(x)$ have a similar meaning with $\alpha$ and $\theta$ in $f(x)$, respectively. The difference is $A(x) \in(0,1]$ and $f(x) \in$ $\left(0, \frac{1}{\sqrt{2 \pi} \theta}\right]$.

There is a limitation that NFN describes fuzzy information by membership degree only. Membership degree alone cannot present information precisely. On the basis of the elicitation of IFS and NFN, Wang et al. [21] proposed the concept of NIFN to overcome the mentioned limitation.

Definition 3 [21]. Let $X$ be a non-empty finite set, $(a, \sigma) \in \tilde{N}$, and $A=\left\langle(a, \sigma), u_{A}, v_{A}\right\rangle$ as a NIFN when its membership function satisfies:

$$
u_{A}(x)=u_{A} e^{-\left(\frac{x-a}{\sigma}\right)^{2}}, x \in X,
$$

and its non-membership function satisfies:

$$
v_{A}(x)=1-\left(1-v_{A}\right) e^{-\left(\frac{x-a}{\sigma}\right)^{2}}, x \in X,
$$

where $0 \leq u_{A}(x) \leq 1,0 \leq v_{A}(x) \leq 1$, and $0 \leq u_{A}(x)+$ $v_{A}(x) \leq 1$. Moreover, $\pi_{A}(x)=1-u_{A}(x)-v_{A}(x)$ is called the hesitance degree of $x$ to $A$. Compared with NFN, NIFN adds the non-membership degree function, which can present the degree of alternatives that do not belong to $(a, \sigma)$. When $u_{A}(x)=1, v_{A}(x)=0$, and NIFN is degenerated to NFN. Therefore, NFN can be regarded as a special case of NIFN. NIFN is graphically shown in Figure 1.

\subsection{Distance of NIFNs}

Distance measure plays a critical role in addressing practical decision problems, and most decision-making models are constructed based on distance measures [55-57]. The distance of NIFNs was defined by Wang and $\mathrm{Li}[58]$ as follows:

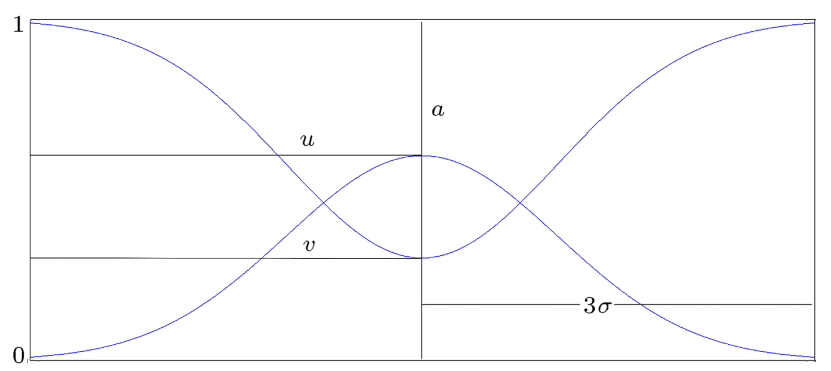

Figure 1. Illustration of Normal Intuitionistic Fuzzy Number (NIFN).
Definition 4 [58]. Let $A_{1}=\left\langle\left(a_{1}, \sigma_{1}\right), u_{1}, v_{1}\right\rangle$ and $A_{2}$ $=\left\langle\left(a_{2}, \sigma_{2}\right), u_{2}, v_{2}\right\rangle$ be two arbitrary NIFNs and then, the Euclidean distance between $A_{1}$ and $A_{2}$ can be defined by Eq. (5) as shown in Box I, when $u_{A}(x)=1$, $v_{A}(x)=0$, and the above distance is reduced to $d\left(A_{1}, A_{2}\right)=\sqrt{\left(a_{1}-a_{2}\right)^{2}+\frac{1}{2}\left(\sigma_{1}-\sigma_{2}\right)^{2}}$, which is the distance of two NFNs.

\subsection{Operations of NIFNs}

Operation is the foundation of information fusion. For aggregating normal intuitionistic fuzzy evaluation information in the decision-making process, Wang et al. [21] proposed the following operations of NIFNs.

Definition 5 [21]. Let $A=\langle(a, \sigma), u, v\rangle, A_{1}=\left\langle\left(a_{1}\right.\right.$, $\left.\left.\sigma_{1}\right), u_{1}, v_{1}\right\rangle$, and $A_{2}=\left\langle\left(a_{2}, \sigma_{2}\right), u_{2}, v_{2}\right\rangle$ be three NIFNs, $\lambda \geq 0$, and then the operations of NIFNs can be defined as follows:

1. $A_{1} \oplus A_{2}=\left\langle\left(a_{1}+a_{2}, \sigma_{1}+\sigma_{2}\right), \frac{\left|a_{1}\right| u_{1}+\left|a_{2}\right| u_{2}}{\left|a_{1}\right|+\left|a_{2}\right|}\right.$,

$$
\left.\frac{\left|a_{1}\right| v_{1}+\left|a_{2}\right| v_{2}}{\left|a_{1}\right|+\left|a_{2}\right|}\right\rangle
$$

when $a=b=0, u_{A_{1}+A_{2}}=\frac{u_{1}+u_{2}}{2}$ and $v_{A_{1}+A_{2}}=\frac{v_{1}+v_{2}}{2}$;

2. $A_{1} \otimes A_{2}=\left\langle\left(a_{1} a_{2}, a_{1} a_{2} \sqrt{\frac{\sigma_{1}^{2}}{a_{1}^{2}}+\frac{\sigma_{2}^{2}}{a_{2}^{2}}}\right), u_{1} u_{2}, v_{1}\right.$

$$
\left.+v_{2}-v_{1} v_{2}\right\rangle
$$

3. $\lambda A=\langle(\lambda a, \lambda \sigma), u, v\rangle$,

4. $A^{\lambda}=\left\langle\left(a^{\lambda}, \lambda^{\frac{1}{2}} a^{\lambda-1} \sigma\right), u^{\lambda}, 1-(1-v)^{\lambda}\right\rangle$,

5. $\frac{1}{A}=\left\langle\left(\frac{1}{a}, \frac{\sigma}{a^{2}}\right), u, v\right\rangle, a \neq 0$.

\section{New comparison method of NIFNs}

In this section, the drawbacks with respect to the existing comparison method of NIFNs are discussed first. An effective ranking method for NIFNs is then proposed.

$$
d\left(A_{1}, A_{2}\right)=\frac{1}{2} \sqrt{\left(\left(1+u_{1}-v_{1}\right) a_{1}-\left(1+u_{2}-v_{2}\right) a_{2}\right)^{2}+\frac{1}{2}\left(\left(1+u_{1}-v_{1}\right) \sigma_{1}-\left(1+u_{2}-v_{2}\right) \sigma_{2}\right)^{2}} .
$$


Comparison method is useful for ranking or selecting different alternatives. Wang et al. [21] proposed the comparison method of NIFNs based on score function and accuracy function as follows:

Definition 6 [21]. Let $A=\langle(a, \sigma), u, v\rangle$ be an arbitrary NIFN and then, the score function of NIFN be defined as follows:

$$
s_{1}(A)=a(u-v) \text { and } s_{2}(A)=\sigma(u-v) .
$$

The accuracy function is defined as:

$$
h_{1}(A)=a(u+v) \text { and } h_{2}(A)=\sigma(u+v) .
$$

Definition 7 [21]. Let $A_{1}=\left\langle\left(a_{1}, \sigma_{1}\right), u_{1}, v_{1}\right\rangle$ and $A_{2}$ $=\left\langle\left(a_{2}, \sigma_{2}\right), u_{2}, v_{2}\right\rangle$ be two arbitrary NIFNs and then, the comparison rules can be defined as follows:

1. if $s_{1}\left(A_{1}\right)>s_{1}\left(A_{2}\right)$, then $A_{1}>A_{2}$;

2. if $s_{1}\left(A_{1}\right)>s_{1}\left(A_{2}\right)$, and $h_{1}\left(A_{1}\right)>h_{1}\left(A_{2}\right)$, then $A_{1}>A_{2}$

3. if $s_{1}\left(A_{1}\right)>s_{1}\left(A_{2}\right)$, and $h_{1}\left(A_{1}\right)=h_{1}\left(A_{2}\right)$ :

(I) when $s_{2}\left(A_{1}\right)<s_{2}\left(A_{2}\right), A_{1}>A_{2}$;

(II) when $s_{2}\left(A_{1}\right)=s_{2}\left(A_{2}\right)$ and $h_{2}\left(A_{1}\right)<h_{2}\left(A_{2}\right)$, $A_{1}>A_{2}$

(III) when $s_{2}\left(A_{1}\right)=s_{2}\left(A_{2}\right)$ and $h_{2}\left(A_{1}\right)=h_{2}\left(A_{2}\right)$, $A_{1}=A_{2}$.

Example 1. Let:

$$
\begin{aligned}
& \phi_{1}=\langle(6,0.6), 0.4,0.2\rangle, \\
& \phi_{2}=\langle(3,0.3), 0.75,0.25\rangle, \\
& \phi_{3}=\langle(6,0.6), 0.6,0.2\rangle, \\
& \phi_{4}=\langle(4,0.4), 0.6,0.3\rangle, \\
& \phi_{5}=\langle(8,0.8), 0.45,0.15\rangle, \\
& \phi_{6}=\langle(5,0.5), 0.45,0.15\rangle,
\end{aligned}
$$

be six NIFNs, and then their score values and accuracy values can be calculated as follows:

$$
\begin{aligned}
& s_{1}\left(\phi_{1}\right)=1.2, \quad s_{1}\left(\phi_{2}\right)=1.5, \quad s_{1}\left(\phi_{3}\right)=2.4, \\
& s_{1}\left(\phi_{4}\right)=1.2, \quad s_{1}\left(\phi_{5}\right)=2.4, \quad \text { and } \quad s_{1}\left(\phi_{6}\right)=1.5 . \\
& s_{2}\left(\phi_{1}\right)=3.6, \quad s_{2}\left(\phi_{2}\right)=3, \quad s_{2}\left(\phi_{3}\right)=4.8, \\
& s_{2}\left(\phi_{4}\right)=3.6, \quad s_{2}\left(\phi_{5}\right)=4.8, \quad \text { and } \quad s_{2}\left(\phi_{6}\right)=3 . \\
& h_{1}\left(\phi_{1}\right)=0.12, \quad h_{1}\left(\phi_{2}\right)=0.15, \quad h_{1}\left(\phi_{3}\right)=0.24, \\
& h_{1}\left(\phi_{4}\right)=0.12, \quad h_{1}\left(\phi_{5}\right)=0.24, \quad \text { and } \quad h_{1}\left(\phi_{6}\right)=0.15 .
\end{aligned}
$$

$$
\begin{aligned}
& h_{2}\left(\phi_{1}\right)=0.36, \quad h_{2}\left(\phi_{2}\right)=0.3, \quad h_{2}\left(\phi_{3}\right)=0.48, \\
& h_{2}\left(\phi_{4}\right)=0.36, \quad h_{2}\left(\phi_{5}\right)=0.48, \quad \text { and } h_{2}\left(\phi_{6}\right)=0.3 .
\end{aligned}
$$

Moreover, according to Definition 7 , there are:

$$
\begin{aligned}
s_{1}\left(\phi_{3}\right) & =s_{1}\left(\phi_{5}\right)>s_{1}\left(\phi_{2}\right)=s_{1}\left(\phi_{6}\right) \\
& >s_{1}\left(\phi_{1}\right)=s_{1}\left(\phi_{4}\right) . \\
s_{2}\left(\phi_{3}\right) & =s_{2}\left(\phi_{5}\right), \quad s_{2}\left(\phi_{2}\right)=s_{2}\left(\phi_{6}\right), \\
s_{2}\left(\phi_{1}\right) & =s_{2}\left(\phi_{4}\right) . \\
h_{1}\left(\phi_{3}\right) & =h_{1}\left(\phi_{5}\right), \quad h_{1}\left(\phi_{2}\right)=h_{1}\left(\phi_{6}\right), \\
h_{1}\left(\phi_{1}\right) & =h_{1}\left(\phi_{4}\right) . \\
h_{2}\left(\phi_{3}\right) & =h_{2}\left(\phi_{5}\right), \quad h_{2}\left(\phi_{2}\right)=h_{2}\left(\phi_{6}\right), \\
h_{2}\left(\phi_{1}\right) & =h_{2}\left(\phi_{4}\right) .
\end{aligned}
$$

Therefore, the ranking can be obtained as $\phi_{3}=\phi_{5} \succ$ $\phi_{2}=\phi_{6} \succ \phi_{4}=\phi_{1}$.

The above comparison method of NIFNs has many non-negligible problems. First, the procedures for identifying the final ranking are tedious; four values need to be computed and four comparisons need to be conducted. Moreover, the ranking obtained by this method is unreasonable in some circumstances. As mentioned in Example 1, it is obvious that $\phi_{3}$ and $\phi_{5}$ are different NIFNs. However, when the above comparison method is used to compare the two NIFNs, the score values and accuracy values of them are always identical, and the ranking is $\phi_{3}=\phi_{5}$. Intuitively, this comparison is unreasonable. The above analysis can also be summarized according to the ranking between $\phi_{2}$ and $\phi_{6}$ as well as $\phi_{4}$ and $\phi_{1}$.

For that matter, it is necessary to explore an appropriate comparison method for NIFNs. Szmidt and Kacprzyk [59] proposed an effective ranking method of IFNs by comprehensively considering the hesitation degree of IFNs and the distance to the positive ideal solution. Motivated by this idea, a valid ranking method of NIFNs is developed to remove the drawbacks in the existing comparison method. The new ranking method systematically integrates the expectation and variance of normal distribution, the hesitation degree of NIFNs, and the distance measure.

Definition 8. Let $\phi_{i}=\left\langle\left(a_{i}, \sigma_{i}\right), u_{i}, v_{i}\right\rangle,(i=1,2, \ldots, n)$ be $n$ arbitrary NIFNs, $\phi^{*}=\left\langle\left(\max \left(a_{i}\right), \min \left(\sigma_{i}\right)\right), 1,0\right\rangle$ be the positive ideal point among $\phi_{i}(i=1,2, \ldots, n)$, $d_{E}\left(\phi^{*}, \phi_{i}\right)$ be the Euclidean distance between $\phi^{*}$ and $\phi_{i}$, and the scoring function $S\left(\phi_{i}\right)$ be defined as follows:

$$
S\left(\phi_{i}\right)=\left(1+\pi_{i}\right) d_{E}\left(\phi^{*}, \phi_{i}\right) .
$$


It is apparent that the smaller the value of $S\left(\phi_{i}\right)$, the better the NIFN $\phi_{i}$.

Example 2. Let $\phi_{1}, \phi_{2}, \phi_{3}, \phi_{4}, \phi_{5}$, and $\phi_{6}$ be the same as those in Example 1 and then, according to Definition 8 , the scoring value $S\left(\phi_{i}\right)$ can be computed as follows:

$$
\begin{aligned}
& S\left(\phi_{1}\right)=6.1603, \quad S\left(\phi_{2}\right)=5.7502, \quad S\left(\phi_{3}\right)=4.5611, \\
& S\left(\phi_{4}\right)=5.9401, \quad S\left(\phi_{5}\right)=3.926, \quad \text { and } \quad S\left(\phi_{6}\right)=6.65 .
\end{aligned}
$$

Therefore, the ranking can be identified as $\phi_{5} \succ \phi_{3} \succ$ $\phi_{2} \succ \phi_{4} \succ \phi_{1} \succ \phi_{6}$.

In conclusion, compared with the existing comparison method of NIFNs, the proposed new ranking method is simple and effective: Only one parameter needs to be computed and the reasonable result can be obtained.

\section{Normal Intuitionistic Power Harmonic (NIPH) aggregation operators}

In this section, based on the operations of NIFNs, three normal intuitionistic generalized aggregation operators are developed by integrating the PA and HM operators. Moreover, some special cases and desirable properties of these operators are discussed.

\subsection{PA operator and HM operator}

To comprehend the subsequent normal intuitionistic generalized aggregation operators easily, the definitions of PA and HM operators are described simply in the following:

Definition 9 [46]. Let $a_{1}, a_{2}, \ldots a_{n}$ be $n$ positive real numbers. The PA operator is a mapping: $R^{n} \rightarrow R$, and it can be defined as follows:

$$
P A\left(a_{1}, a_{2}, \ldots, a_{n}\right)=\frac{\sum_{i=1}^{n}\left(1+T\left(a_{i}\right)\right) a_{i}}{\sum_{i=1}^{n}\left(1+T\left(a_{i}\right)\right)},
$$

where $T\left(a_{i}\right)=\sum_{j=1, i \neq j}^{n} \operatorname{Sup}\left(a_{i}, a_{j}\right)$, and $\operatorname{Sup}\left(a_{i}, a_{j}\right)$ is the support degree of $a_{i}$ from $a_{j}$.
The support degree satisfies the following properties:

1. $\operatorname{Sup}\left(a_{i}, a_{j}\right) \in[0,1]$;

2. $\operatorname{Sup}\left(a_{i}, a_{j}\right)=\operatorname{Sup}\left(a_{j}, a_{i}\right)$;

3. $\operatorname{Sup}\left(a_{i}, a_{j}\right)>\operatorname{Sup}(x, y)$ if $d\left(a_{i}, a_{j}\right)<d(x, y)$, where $d\left(a_{i}, a_{j}\right)$ indicates the distance between $a_{i}$ and $a_{j}$.

Definition 10 [50]. For $n$ positive real numbers $a_{1}$, $a_{2}, \ldots, a_{n}$, the HM operator can be defined as follows:

$$
H M\left(a_{1}, a_{2}, \ldots, a_{n}\right)=\frac{1}{\sum_{i=1}^{n} \frac{1}{a_{i}}} .
$$

\subsection{Normal Intuitionistic Generalized Weighted Power Harmonic Operator (NIGWPH)}

In this subsection, based on the weighted arithmetic averaging, PA, and HM operators, the generalized weighted power harmonic operator under normal intuitionistic fuzzy environment is investigated. The definition of the Normal Intuitionistic Generalized Weighted Power Harmonic (NIGWPH) operator and its corresponding theorems are provided below:

Definition 11. Let $A_{i}=\left\langle\left(a_{i}, \sigma_{i}\right), u_{i}, v_{i}\right\rangle, \quad(i=1$, $2, \ldots, n)$ be a collection of NIFNs and $w_{i}(i=1$, $2, \ldots, n)$ be the weight of $A(i=1,2, \ldots, n)$, with $w_{i} \in$ $[0,1]$, and $\sum_{i=1}^{n} w_{i}=1$, and then the NIGWPH operator can be defined by Eq. (9) as shown in Box II, where $\lambda \in(-\infty, 0) \cup(0,+\infty)$, and $T\left(A_{i}\right)=$ $\sum_{j=1, i \neq j}^{n} w_{j} \operatorname{Sup}\left(A_{i}, A_{j}\right)$ is the comprehensive weighted support degree of $A_{i}$ from $A_{j}(i \neq j, j=1,2, \ldots, n)$. Based on the operations of NIFNs, the specific expression of NIGWPH can be given below:

Theorem 1. Let $A_{i}=\left\langle\left(a_{i}, \sigma_{i}\right), u_{i}, v_{i}\right\rangle,(i=1,2, \ldots, n)$ be a collection of NIFNs and $w_{i}(i=1,2, \ldots, n)$ be the weight of $A_{i}(i=1,2, \ldots, n)$, with $w_{i} \in[0,1]$ and $\sum_{i=1}^{n} w_{i}=1$, and then the aggregated value obtained by the NIGWPH operator is also an NIFN and:

$$
\begin{array}{rl}
N I G W P & H\left(A_{1}, A_{2}, \ldots, A_{n}\right) \\
& =\frac{1}{\left(\frac{w_{1}\left(1+T\left(A_{1}\right)\right)}{\sum_{i=1}^{n} w_{i}\left(1+T\left(A_{i}\right)\right)}\left(\frac{1}{A_{1}}\right)^{\lambda} \oplus \frac{w_{2}\left(1+T\left(A_{2}\right)\right)}{\sum_{i=1}^{n} w_{i}\left(1+T\left(A_{i}\right)\right)}\left(\frac{1}{A_{2}}\right)^{\lambda} \oplus \cdots \oplus \frac{w_{n}\left(1+T\left(A_{n}\right)\right)}{\sum_{i=1}^{n} w_{i}\left(1+T\left(A_{i}\right)\right)}\left(\frac{1}{A_{n}}\right)^{\lambda}\right)^{\frac{1}{\lambda}}} \\
& =\frac{1}{\left(\sum_{i=1}^{n} \frac{w_{i}\left(1+T\left(A_{i}\right)\right)}{\sum_{i=1}^{n} w_{i}\left(1+T\left(A_{i}\right)\right)}\left(\frac{1}{A_{i}}\right)^{\lambda}\right)^{\frac{1}{\lambda}}} \cdot
\end{array}
$$




$$
\begin{array}{rl}
N I G W & P H\left(A_{1}, A_{2}, \ldots, A_{n}\right) \\
= & \left\langle\left(\frac{1}{\left(\sum_{i=1}^{n} \frac{\gamma_{i}}{a_{i}^{\lambda}}\right)^{\frac{1}{\lambda}}}, \frac{\sum_{i=1}^{n} \frac{\sigma_{i} \gamma_{i}}{a_{i}^{\lambda}+1}}{\left(\sum_{i=1}^{n} \frac{\gamma_{i}}{a_{i}^{\lambda}}\right)^{\frac{1}{\lambda}+1}}\right),\right. \\
& \left(\frac{\sum_{i=1}^{n} \frac{\gamma_{i} u_{i}^{\lambda}}{\left|a_{i}\right|^{\lambda}}}{\sum_{i=1}^{n} \frac{\gamma_{i}}{\left|a_{i}\right|^{\lambda}}}\right)^{\frac{1}{\lambda}}, 1 \\
& \left.-\left(\frac{\sum_{i=1}^{n} \frac{\gamma_{i}}{\left|a_{i}\right|^{\lambda}}\left(1-v_{i}\right)^{\lambda}}{\sum_{i=1}^{n} \frac{\gamma_{i}}{\left|a_{i}\right|^{\lambda}}}\right)^{\frac{1}{\lambda}}\right\rangle
\end{array}
$$

where $\gamma_{i}=\frac{w_{i}\left(1+T\left(A_{i}\right)\right)}{\sum_{i=1}^{n} w_{i}\left(1+T\left(A_{i}\right)\right)}$ and $T\left(A_{i}\right)$ is the comprehensive weighted support degree of $A_{i}$.

Proof. Obviously, according to Definition 3, the above aggregated value obtained by the NIGWPH operator is also an NIFN. Moreover, Eq. (10) can be proved easily by mathematical induction on $n$ as follows:

1. For $n=2$, since:

$$
\frac{w_{1}\left(1+T\left(A_{1}\right)\right)}{\sum_{i=1}^{n} w_{i}\left(1+T\left(A_{i}\right)\right)}\left(\frac{1}{A_{1}}\right)^{\lambda}
$$

$$
\begin{aligned}
& =\left\langle\left(\gamma_{1}\left(\frac{1}{a_{1}}\right)^{\lambda}, \gamma_{1} \lambda^{\frac{1}{2}} \frac{\sigma_{1}}{a_{1}^{\lambda+1}}\right), u_{1}^{\lambda}, 1-\left(1-v_{1}\right)^{\lambda}\right\rangle, \\
& \frac{w_{2}\left(1+T\left(A_{2}\right)\right)}{\sum_{i=1}^{n} w_{i}\left(1+T\left(A_{i}\right)\right)}\left(\frac{1}{A_{2}}\right)^{\lambda} \\
& =\left\langle\left(\gamma_{2}\left(\frac{1}{a_{2}}\right)^{\lambda}, \gamma_{2} \lambda^{\frac{1}{2}} \frac{\sigma_{2}}{a_{2}^{\lambda+1}}\right), u_{2}^{\lambda},\right. \\
& \left.1-\left(1-v_{2}\right)^{\lambda}\right\rangle .
\end{aligned}
$$

Then, we have the equation shown in Box III.

2. If Eq. (10) holds for $n=k$, then the equation shown in Box IV is obtained. When $n=k+1$, according to the operations of INFNs, we have the equation shown in Box V. That is, Eq. (10) also holds for $n=k+1$. Therefore, Eq. (10) is true for all $n$ 's.

Theorem 2 (Idempotency). Let $A_{i}=\left\langle\left(a_{i}, \sigma_{i}\right), u_{i}\right.$, $\left.v_{i}\right\rangle,(i=1,2, \ldots, n)$ be a collection of NIFNs. If $A_{i}$ $=\tilde{A}$ for all $i=1,2, \ldots, n$ and, then, NIGWPH $\left(A_{1}, A_{2}, \ldots, A_{n}\right)=\tilde{A}$.

Proof. Since $A_{i}=\tilde{A}(i=1,2, \ldots, n)$ based on the

$$
\begin{array}{rl}
N I G W & P H\left(A_{1}, A_{2}\right)=\frac{1}{\left(\frac{w_{1}\left(1+T\left(A_{1}\right)\right)}{\sum_{i=1}^{n} w_{i}\left(1+T\left(A_{i}\right)\right)}\left(\frac{1}{A_{1}}\right)^{\lambda} \oplus \frac{w_{2}\left(1+T\left(A_{2}\right)\right)}{\sum_{i=1}^{n} w_{i}\left(1+T\left(A_{i}\right)\right)}\left(\frac{1}{A_{2}}\right)^{\lambda}\right)^{\frac{1}{\lambda}}} \\
& =\left\langle\left(\frac{1}{\left(\frac{\gamma_{1}}{a_{1}^{\lambda}}+\frac{\gamma_{2}}{a_{2}^{\lambda}}\right)^{\frac{1}{\lambda}},}, \frac{\frac{\sigma_{1} \gamma_{1}}{a_{1}^{\lambda}+1}+\frac{\sigma_{2} \gamma_{2}}{a_{2}^{\lambda+1}}}{\left(\frac{\gamma_{1}}{a_{1}^{\lambda}}+\frac{\gamma_{2}}{a_{2}^{\lambda}}\right)^{\frac{1}{\lambda}+1}}\right),\left(\frac{\frac{\gamma_{1} u_{1}^{\lambda}}{\left|a_{1}\right|^{\lambda}}+\frac{\gamma_{2} u_{2}^{\lambda}}{\left|a_{2}\right|^{\lambda}}}{\mid \frac{1}{\left|a_{1}\right|^{\lambda}}+\frac{\gamma_{2}}{\left|a_{2}\right|^{\lambda}}}\right)^{\frac{1}{\lambda}}, 1-\left(\frac{\frac{\gamma_{1}}{\left|a_{1}\right|^{\lambda}}\left(1-v_{1}\right)^{\lambda}+\frac{\gamma_{2}}{\left|a_{2}\right|^{\lambda}}\left(1-v_{2}\right)^{\lambda}}{\frac{\gamma_{1}}{\left|a_{1}\right|^{\lambda}}+\frac{\gamma_{2}}{\left|a_{2}\right|^{\lambda}}}\right) .\right.
\end{array}
$$

\section{Box III}

$N I G W P H\left(A_{1}, A_{2}, \ldots, A_{k}\right)$

$$
\begin{aligned}
& =\frac{1}{\left(\frac{w_{1}\left(1+T\left(A_{1}\right)\right)}{\sum_{i=1}^{k} w_{i}\left(1+T\left(A_{i}\right)\right)}\left(\frac{1}{A_{1}}\right)^{\lambda} \oplus \frac{w_{2}\left(1+T\left(A_{2}\right)\right)}{\sum_{i=1}^{k} w_{i}\left(1+T\left(A_{i}\right)\right)}\left(\frac{1}{A_{2}}\right)^{\lambda} \oplus \cdots \oplus \frac{w_{k}\left(1+T\left(A_{k}\right)\right)}{\sum_{i=1}^{k} w_{i}\left(1+T\left(A_{i}\right)\right)}\left(\frac{1}{A_{k}}\right)^{\lambda}\right)^{\frac{1}{\lambda}}} \\
& =\left\langle\left(\frac{1}{\left(\sum_{i=1}^{k} \frac{\gamma_{i}}{a_{i}^{\lambda}}\right)^{\frac{1}{\lambda}}}, \frac{\sum_{i=1}^{k} \frac{\sigma_{i} \gamma_{i}}{a_{i}^{\lambda+1}}}{\left(\sum_{i=1}^{k} \frac{\gamma_{i}}{a_{i}^{\lambda}}\right)^{\frac{1}{\lambda}+1}}\right),\left(\frac{\sum_{i=1}^{k} \frac{\gamma_{i} u_{i}^{\lambda}}{\left|a_{i}\right|^{\lambda}}}{\sum_{i=1}^{k} \frac{\gamma_{i}}{\left|a_{i}\right|^{\lambda}}}\right), 1-\left(\frac{\sum_{i=1}^{k} \frac{\gamma_{i}}{\left|a_{i}\right|^{\lambda}}\left(1-v_{i}\right)^{\lambda}}{\sum_{i=1}^{k} \frac{\gamma_{i}}{\left|a_{i}\right|^{\lambda}}}\right)\right\rangle .
\end{aligned}
$$




$$
\begin{array}{rl}
N I G W & P H\left(A_{1}, A_{2}, \ldots, A_{k}, A_{k+1}\right) \\
= & \frac{1}{\left(\sum_{i=1}^{k} \frac{w_{i}\left(1+T\left(A_{i}\right)\right)}{\sum_{i=1}^{k+1} w_{i}\left(1+T\left(A_{i}\right)\right)}\left(\frac{1}{A_{i}}\right)^{\lambda} \oplus \frac{w_{k+1}\left(1+T\left(A_{k+1}\right)\right)}{\sum_{i=1}^{k+1} w_{i}\left(1+T\left(A_{i}\right)\right)}\left(\frac{1}{A_{k+1}}\right)^{\lambda}\right)^{\frac{1}{\lambda}}} \\
= & \left\langle\left(\frac{1}{\left(\sum_{i=1}^{k+1} \frac{\gamma_{i}}{a_{i}^{\lambda}}\right)^{\frac{1}{\lambda}}}, \frac{\sum_{i=1}^{k+1} \frac{\sigma_{i} \gamma_{i}}{a_{i}^{\lambda+1}}}{\left(\sum_{i=1}^{k+1} \frac{\gamma_{i}}{a_{i}^{\lambda}}\right)^{\frac{1}{\lambda}+1}}\right),\left(\frac{\sum_{i=1}^{k+1} \frac{\gamma_{i} u_{i}^{\lambda}}{\left|a_{i}\right|^{\lambda}}}{\sum_{i=1}^{k+1} \frac{\gamma_{i}}{\left|a_{i}\right|^{\lambda}}}\right), 1-\left(\frac{\sum_{i=1}^{k+1} \frac{\gamma_{i}}{\left|a_{i}\right|^{\lambda}}\left(1-v_{i}\right)^{\lambda}}{\sum_{i=1}^{k+1} \frac{\gamma_{i}}{\left|a_{i}\right|^{\lambda}}}\right)^{\frac{1}{\lambda}}\right\rangle .
\end{array}
$$

operations of NIFNs and Definition 11, there is:

$$
\begin{aligned}
N I G W P H\left(A_{1}, A_{2}, \ldots, A_{n}\right) \\
=\frac{1}{\left(\sum_{i=1}^{n} \frac{w_{i}\left(1+T\left(A_{i}\right)\right)}{\sum_{i=1}^{n} w_{i}\left(1+T\left(A_{i}\right)\right)}\left(\frac{1}{A_{i}}\right)^{\lambda}\right)^{\frac{1}{\lambda}}} \\
=\frac{1}{\left(\sum_{i=1}^{n} \frac{w_{i}(1+T(\tilde{A}))}{\sum_{i=1}^{n} w_{i}(1+T(\tilde{A}))}\left(\frac{1}{\tilde{A}}\right)^{\lambda}\right)^{\frac{1}{\lambda}}}=\tilde{A} .
\end{aligned}
$$

Theorem 3 (Commutativity). Let $A_{i}=\left\langle\left(a_{i}, \sigma_{i}\right)\right.$, $\left.u_{i}, v_{i}\right\rangle,(i=1,2, \ldots, n)$ be a collection of NIFNs, and $\left(A_{1}^{\prime}, A_{2}^{\prime}, \cdots, A_{n}^{\prime}\right)$ be any permutation of $\left(A_{1}\right.$, $\left.A_{2}, \cdots, A_{n}\right)$. If the weight of is not relevant to the position of $A_{i}$ variables, then:

$$
\begin{aligned}
& N I G W P H\left(A_{1}, A_{2}, \ldots, A_{n}\right) \\
& =N I G W P H\left(A_{1}^{\prime}, A_{2}^{\prime}, \cdots, A_{n}^{\prime}\right) .
\end{aligned}
$$

The support degree of $A_{i}$ is determined by the distance measure between $A_{i}$ and $A_{j}(j=1,2, \ldots, n, j \neq$ $i$ ), and will not be affected by its position in the permutation. Thus, Theorem 3 can be easily proven.

In general, there are four kinds of basic properties (idempotency, commutativity, monotonicity and boundedness) for fuzzy aggregation operators. However, different operators have distinct features, and some operators can satisfy all of these properties and some operators can satisfy some of these properties. It is noted that it is difficult to consider the monotonicity, and boundedness of the NIGWPH operator because the support degree will change and must be recalculated when the input variables vary.

Some special cases of the NIGWPH operator are described as follows:

1. If $w=(1 / n, 1 / n, \ldots, 1 / n)$, then the NIGWPH operator is degenerated to the Normal Intuitionistic
Generalized Power Harmonic (NIGPH) operator as follows:

$$
\begin{aligned}
& N I G P H\left(A_{1}, A_{2}, \ldots, A_{n}\right) \\
& =\frac{1}{\left(\sum_{i=1}^{n} \frac{\left(1+T\left(A_{i}\right)\right)}{\sum_{i=1}^{n}\left(1+T\left(A_{i}\right)\right)}\left(\frac{1}{A_{i}}\right)^{\lambda}\right)^{\frac{1}{\lambda}}} \\
& =\left\langle\left(\frac{1}{\left(\sum_{i=1}^{n} \frac{\rho_{i}}{a_{i}^{\lambda}}\right)^{\frac{1}{\lambda}}}, \frac{\sum_{i=1}^{n} \rho_{i} \frac{\sigma_{i}}{a_{i}^{\lambda+1}}}{\left(\sum_{i=1}^{n} \frac{\rho_{i}}{a_{i}^{\lambda}}\right)^{\frac{1}{\lambda}+1}}\right),\right. \\
& \left(\frac{\sum_{i=1}^{n} \frac{\rho_{i} u_{i}^{\lambda}}{\left|a_{i}\right|^{\lambda}}}{\sum_{i=1}^{n} \frac{\rho_{i}}{\left|a_{i}\right|^{\lambda}}}\right)^{\frac{1}{\lambda}}, 1 \\
& \left.-\left(\frac{\sum_{i=1}^{n} \frac{\rho_{i}}{\left|a_{i}\right|^{\lambda}}\left(1-v_{i}\right)^{\lambda}}{\sum_{i=1}^{n} \frac{\rho_{i}}{\left|a_{i}\right|^{\lambda}}}\right)^{\frac{1}{\lambda}}\right\rangle .
\end{aligned}
$$

It is worth noting that the NIGPH operator is capable of capturing the weight of $A_{i}(i=1,2, \ldots, n)$ as $\rho_{i}=\frac{\left(1+T\left(A_{i}\right)\right)}{\sum_{i=1}^{n}\left(1+T\left(A_{i}\right)\right)}(i=1,2, \ldots, n)$ according to the support degree among different variables.

2. If $\lambda=1$, then the NIGWPH operator is reduced to the Normal Intuitionistic Weighted Power Harmonic (NIWPH) operator as:

$$
\begin{array}{rl}
N I W & P H\left(A_{1}, A_{2}, \ldots, A_{n}\right) \\
& =\frac{1}{\sum_{i=1}^{n} \frac{w_{i}\left(1+T\left(A_{i}\right)\right)}{\sum_{i=1}^{n} w_{i}\left(1+T\left(A_{i}\right)\right)} \frac{1}{A_{i}}} \\
& =\left\langle\left(\frac{1}{\sum_{i=1}^{n} \frac{\gamma_{i}}{a_{i}}}, \frac{\sum_{i=1}^{n} \frac{\gamma_{i} \sigma_{i}}{a_{i}^{2}}}{\left(\sum_{i=1}^{n} \frac{\gamma_{i}}{a_{i}}\right)^{2}}\right), \frac{\sum_{i=1}^{n} \frac{\gamma_{i} u_{i}}{\left|a_{i}\right|}}{\sum_{i=1}^{n} \frac{\gamma_{i}}{\left|a_{i}\right|}}\right. \\
& \left.\frac{\sum_{i=1}^{n} \frac{\gamma_{i} v_{i}}{\left|a_{i}\right|}}{\sum_{i=1}^{n} \frac{\gamma_{i}}{\left|a_{i}\right|}}\right\rangle .
\end{array}
$$


3. If $\lambda \rightarrow 0$, then the NIGWPH operator becomes the Normal Intuitionistic Weighted Geometric Power Harmonic (NIWGPH) operator as follows:

$$
\begin{aligned}
N I W & \operatorname{PPH}\left(A_{1}, A_{2}, \ldots, A_{n}\right) \\
& =\frac{1}{\prod_{i=1}^{n}\left(\frac{1}{A_{i}}\right)^{\frac{w_{i}\left(1+T\left(A_{i}\right)\right)}{\sum_{i=1}^{n} w_{i}\left(1+T\left(A_{i}\right)\right)}}} \\
& =\left\langle\left(\frac{1}{\prod_{i=1}^{n}\left(\frac{1}{a_{i}}\right)^{\gamma_{i}}}, \frac{\sqrt{\sum_{i=1}^{n} \frac{\gamma_{i} \sigma_{i}^{2}}{a_{i}^{2}}}}{\prod_{i=1}^{n}\left(\frac{1}{a_{i}}\right)^{\gamma_{i}}}\right),\right. \\
& \left.\prod_{i=1}^{n} u_{i}^{\gamma_{i}}, 1-\prod_{i=1}^{n}\left(1-v_{i}\right)^{\gamma_{i}}\right\rangle .
\end{aligned}
$$

4. If $\lambda=-1$, then the NIGWPH operator becomes the Normal Intuitionistic Weighted Power (NIWP) operator as:

$$
\begin{array}{rl}
N I W & P\left(A_{1}, A_{2}, \ldots, A_{n}\right) \\
& =\sum_{i=1}^{n} \frac{w_{i}\left(1+T\left(A_{i}\right)\right)}{\sum_{i=1}^{n} w_{i}\left(1+T\left(A_{i}\right)\right)} A_{i} \\
& =\left\langle\left(\sum_{i=1}^{n} a_{i} \gamma_{i}, \sum_{i=1}^{n} \sigma_{i} \gamma_{i}\right),\right. \\
& \left.\frac{\sum_{i=1}^{n}\left|a_{i}\right| \gamma_{i}}{\sum_{i=1}^{n} \frac{\left|a_{i}\right| \gamma_{i}}{u_{i}}}, 1-\frac{\sum_{i=1}^{n}\left|a_{i}\right| \gamma_{i}}{\sum_{i=1}^{n} \frac{\left|a_{i}\right| \gamma_{i}}{1-v_{i}}}\right\rangle
\end{array}
$$

\subsection{Normal intuitionistic generalized induced \\ ordered weighted power harmonic operator}

In this subsection, the generalized induced ordered weighted power harmonic aggregation operator under normal intuitionistic fuzzy situation is developed, which is an integration of Induced Ordered Weighted Averaging (IOWA), PA, and HM operators. The definition of the Normal Intuitionistic Generalized Induced Ordered Weighted Power Harmonic (NIGIOWPH) operator and its relevant theorems are presented in the following.

Definition 12. Let $A_{i}=\left\langle\left(a_{i}, \sigma_{i}\right), u_{i}, v_{i}\right\rangle,(i=1,2$, $\ldots, n)$ be a collection of NIFNs and then, the NIGIOWPH operator can be defined as follows:

$$
\begin{aligned}
& N I G \operatorname{IOWPH}\left(\left\langle u_{1}, A_{1}\right\rangle,\left\langle u_{2}, A_{2}\right\rangle, \ldots,\left\langle u_{n}, A_{n}\right\rangle\right) \\
& =\frac{1}{\left(\sum_{i=1}^{n} \kappa_{i}\left(\frac{1}{\tilde{A}_{\text {index }(i)}}\right)^{\lambda}\right)^{\frac{1}{\lambda}}}
\end{aligned}
$$

where $\lambda \in(-\infty, 0) \cup(0,+\infty),\left\langle u_{i}, A_{i}\right\rangle$ is the 2 -tuple with ordered induced variable $u_{i}$ and NIFN $A_{i}$ (index(1), index $(2), \ldots, \operatorname{index}(n))$ is a permutation of $(1,2, \ldots, n)$ such that $u_{\text {index }(i-1)} \geq u_{\text {index }(i)}$ for all $i=1,2,3, \ldots, n$, $\left\langle u_{\text {index }(i)}, \tilde{A}_{\text {index }(i)}\right\rangle$ is the 2 -tuple with the $i$-th largest ordered induced variable $u_{i}$ in $\left\{u_{1}, u_{2}, \ldots, u_{n}\right\}$ and the corresponding NIFN $A_{i}$ in $\left\langle u_{i}, A_{i}\right\rangle$, and $\kappa_{i}$ is the associated weight that satisfies the following formulation:

$$
\begin{aligned}
& T\left(\tilde{A}_{i \operatorname{index}(i)}\right)=\sum_{j=1, j \neq i}^{n} \operatorname{Sup}\left(\tilde{A}_{i n d e x(i)}, \tilde{A}_{i n d e x(j)}\right), \\
& R_{i}=\sum_{j=1}^{i} V_{i n d e x(j)}, \\
& T V=\sum_{i=1}^{n} V_{i n d e x(i)}, \quad V_{i n d e x(i)}=1+T\left(\tilde{A}_{i n d e x(i)}\right), \\
& \kappa_{i}=g\left(\frac{R_{i}}{T V}\right)-g\left(\frac{R_{i-1}}{T V}\right),
\end{aligned}
$$

where the function $g:[0,1] \rightarrow[0,1]$ is a Basic UnitInterval Monotone (BUM) function satisfying $g(0)=0$, $g(1)=1$, and $g(x) \geq g(y)$, if $x>y$. Similarly, $\operatorname{Sup}\left(\tilde{A}_{\text {index }(i)}, \tilde{A}_{\text {index }(j)}\right)$ indicates the support degree of the $i$ th largest argument $\tilde{A}_{\text {index }(i)}$ from $\tilde{A}_{i n d e x(j)}$, and $T\left(A_{i}\right)$ is the comprehensive support degree of $\tilde{A}_{\text {index }(i)}$.

In the NIGIOWPH operator, the reordering of the NIFN aggregated values $\left\{A_{1}, A_{2}, \ldots, A_{n}\right\}$ is induced by the order of $\left\{u_{1}, u_{2}, \ldots, u_{n}\right\}$. If the reordering of the NIFN aggregated values $\left\{A_{1}, A_{2}, \ldots, A_{n}\right\}$ is induced by the order of $\left\{A_{1}, A_{2}, \ldots, A_{n}\right\}$ (namely, $u_{i}=A_{i}(i=$ $1,2, \ldots, n))$, then the NIGIOWPH operator becomes the Normal Intuitionistic Generalized Ordered Weighted Power Harmonic (NIGOWPH) operator

Based on the operations of NIFNs, the expression of the NIGIOWPH operator can be given below.

Theorem 4. Let $A_{i}=\left\langle\left(a_{i}, \sigma_{i}\right), u_{i}, v_{i}\right\rangle,(i=1,2, \ldots, n)$ be a collection of NIFNs, and then the aggregated value obtained by the NIGIOWPH operator is given below:

$$
\begin{aligned}
& N I G I O W P H\left(\left\langle u_{1}, A_{1}\right\rangle,\left\langle u_{2}, A_{2}\right\rangle, \ldots,\left\langle u_{n}, A_{n}\right\rangle\right)
\end{aligned}
$$

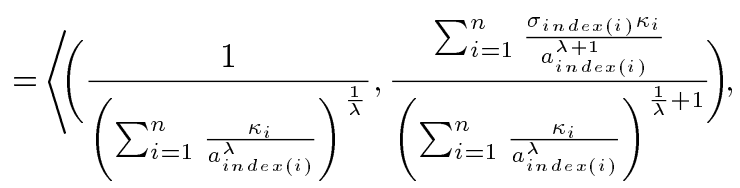

$$
\begin{aligned}
& \left(\frac{\sum_{i=1}^{n} \frac{\kappa_{i} u_{i n d e x(i)}^{\lambda}}{\left|a_{i n d e x(i)}\right|^{\lambda}}}{\sum_{i=1}^{n} \frac{\kappa_{i}}{\left|a_{i n d e x(i)}\right|^{\lambda}}}\right)^{\frac{1}{\lambda}}, 1 \\
& \left.-\left(\frac{\sum_{i=1}^{n} \frac{\kappa_{i}}{\left|a_{\text {index }(i)}\right|^{\lambda}}\left(1-v_{\text {index }(i))^{\lambda}}\right.}{\sum_{i=1}^{n} \frac{\kappa_{i}}{\left|a_{\text {index }(i)}\right|^{\lambda}}}\right)\right\rangle .
\end{aligned}
$$


The aggregated value obtained by the NIGIOWPH operator is also an NIFN, and Eq. (16) can be proven easily through mathematical induction on $n$.

Theorem 5 (Idempotency). Let $A_{i}=\left\langle\left(a_{i}, \sigma_{i}\right), u_{i}\right.$, $\left.v_{i}\right\rangle,(i=1,2, \ldots, n)$ be a collection of NIFNs. If $A_{i}$ $=\tilde{A}$ for all $i=1,2, \ldots, n$ and then, NIGIOWPH $\left(\left\langle u_{1}, A_{1}\right\rangle,\left\langle u_{2}, A_{2}\right\rangle, \ldots,\left\langle u_{n}, A_{n}\right\rangle\right)=\tilde{A}$.

Theorem 6 (Commutativity). Let $\left(A_{1}^{\prime}, A_{2}^{\prime}, \ldots, A_{n}^{\prime}\right)$ be any permutation of $\left(A_{1}, A_{2}, \ldots, A_{n}\right)$ and:

$$
\begin{aligned}
& N \operatorname{NIGIOWPH}\left(\left\langle u_{1}, A^{\prime}{ }_{1}\right\rangle,\left\langle u_{2}, A_{2}^{\prime}\right\rangle, \ldots,\left\langle u_{n}, A_{n}^{\prime}\right\rangle\right) \\
& \quad=N I G I O W P H\left(\left\langle u_{1}, A_{1}\right\rangle,\left\langle u_{2}, A_{2}\right\rangle, \ldots,\left\langle u_{n}, A_{n}\right\rangle\right) .
\end{aligned}
$$

Theorem 5 can be easily proven based on Theorem 2 . Moreover, Theorem 6 is apparently true because all input variables will be reordered using the IOWA operator.

Theorem 7. If $g(x)=x$, then the NIGIOWPH operator is reduced to the NIGPH operator, that is:

$$
\begin{aligned}
& \operatorname{NIGIOWPH}\left(\left\langle u_{1}, A_{1}\right\rangle,\left\langle u_{2}, A_{2}\right\rangle, \ldots,\left\langle u_{n}, A_{n}\right\rangle\right) \\
& \quad=\operatorname{NIGPH}\left(A_{1}, A_{2}, \ldots, A_{n}\right) .
\end{aligned}
$$

Proof. If $g(x)=x$, then:

$$
\kappa_{i}=\frac{R_{i}-R_{i-1}}{T V}=\frac{1+T\left(\tilde{A}_{i n d e x(i)}\right)}{\sum_{i=1}^{n} 1+T\left(\tilde{A}_{i n d e x(i)}\right)},
$$

and there are:

$$
\begin{aligned}
& N I G I O W P H\left(\left\langle u_{1}, A_{1}\right\rangle,\left\langle u_{2}, A_{2}\right\rangle, \ldots,\left\langle u_{n}, A_{n}\right\rangle\right) \\
& =\frac{1}{\left(\sum_{i=1}^{n} \frac{1+T\left(\tilde{A}_{\text {index }(i)}\right)}{\sum_{i=1}^{n} 1+T\left(\tilde{A}_{\text {index }(i)}\right)}\left(\frac{1}{\tilde{A}_{\text {index }(i)}}\right)^{\lambda}\right)^{\frac{1}{\lambda}}} \\
& =\frac{1}{\left(\sum_{i=1}^{n} \frac{1+T\left(A_{i}\right)}{\sum_{i=1}^{n}\left(1+T\left(A_{i}\right)\right)}\left(\frac{1}{A_{i}}\right)^{\lambda}\right)^{\frac{1}{\lambda}}} \\
& =N I G P H\left(A_{1}, A_{2}, \ldots, A_{n}\right) .
\end{aligned}
$$

The NIGIOWPH operator has special cases when the certain value of $\lambda$ is given:

1. If $\lambda=1$, then the NIGIOWPH operator is reduced to the Normal Intuitionistic Induced Ordered Weighted Power Harmonic (NIIOWPH) operator as:

$$
\begin{aligned}
& N I I O W P H\left(\left\langle u_{1}, A_{1}\right\rangle,\left\langle u_{2}, A_{2}\right\rangle, \ldots,\left\langle u_{n}, A_{n}\right\rangle\right) \\
& =\frac{1}{\sum_{i=1}^{n} \kappa_{i} \frac{1}{\tilde{A}_{\text {index }(i)}}} \\
& =\left\langle\left(\frac{1}{\sum_{i=1}^{n} \frac{\kappa_{i}}{a_{i n d e x(i)}}}, \frac{\sum_{i=1}^{n} \frac{\kappa_{i} \sigma_{i n d e x(i)}}{a_{i n d e x(i)}^{2}}}{\left(\sum_{i=1}^{n} \frac{\kappa_{i}}{a_{i n d e x(i)}}\right)^{2}}\right),\right. \\
& \left.\frac{\sum_{i=1}^{n} \frac{\kappa_{i} u_{i n d e x(i)}}{\left|a_{i n d e x(i)}\right|}}{\sum_{i=1}^{n} \frac{\kappa_{i}}{\left|a_{i n d e x(i)}\right|}}, \frac{\sum_{i=1}^{n} \frac{\kappa_{i} v_{i n d e x}(i)}{\left|a_{i n d e x(i)}\right|}}{\sum_{i=1}^{n} \frac{\kappa_{i}}{\left|a_{\text {index }(i)}\right|}}\right\rangle .
\end{aligned}
$$

2. If $\lambda \rightarrow 0$, then the NIGIOWPH operator becomes the Normal Intuitionistic Induced Ordered Weighted Geometric Power Harmonic (NIIOWGPH) operator as follows:

$$
\begin{aligned}
N & \operatorname{IIOWGPH}\left(\left\langle u_{1}, A_{1}\right\rangle,\left\langle u_{2}, A_{2}\right\rangle, \ldots,\left\langle u_{n}, A_{n}\right\rangle\right) \\
= & \frac{1}{\prod_{i=1}^{n}\left(\frac{1}{\tilde{A}_{\text {index }(i)}}\right)^{\kappa_{i}}} \\
= & \left\langle\left(\frac{1}{\prod_{i=1}^{n}\left(\frac{1}{a_{\text {index }(i)}}\right)^{\kappa_{i}}}, \frac{\sqrt{\sum_{i=1}^{n} \frac{\kappa_{i} \sigma_{\text {index }(i)}^{2}}{a_{\text {index }(i)}^{2}}}}{\prod_{i=1}^{n}\left(\frac{1}{a_{\text {index }(i)}}\right)^{\kappa_{i}}}\right),\right. \\
& \prod_{i=1}^{n} u_{i n d e x(i)}^{\kappa_{i}}, 1 \\
& \left.-\prod_{i=1}^{n}\left(1-v_{\text {index }(i)}\right)^{\kappa_{i}}\right\rangle .
\end{aligned}
$$

3. If $\lambda=-1$, then the NIGIOWPH operator is reduced to the Normal Intuitionistic Induced Ordered Weighted Power (NIIOWP) operator as:

$$
\begin{aligned}
& N I I O W P\left(\left\langle u_{1}, A_{1}\right\rangle,\left\langle u_{2}, A_{2}\right\rangle, \ldots,\left\langle u_{n}, A_{n}\right\rangle\right) \\
& =\sum_{i=1}^{n} \kappa_{i} \tilde{A}_{i n \operatorname{dex}(i)} \\
& =\left\langle\left(\sum_{i=1}^{n} a_{i n d e x(i)} \kappa_{i}, \sum_{i=1}^{n} \sigma_{i n d e x(i)} \kappa_{i}\right),\right. \\
& \left.\frac{\sum_{i=1}^{n} a_{i n d e x(i)} \kappa_{i}}{\sum_{i=1}^{n} \frac{\left|a_{\text {index }(i)}\right| \kappa_{i}}{u_{\text {index(i) }}}}, 1-\frac{\sum_{i=1}^{n} a_{i n d e x(i)} \kappa_{i}}{\sum_{i=1}^{n} \frac{\left|a_{\text {index }(i)}\right| \kappa_{i}}{1-v_{\text {index }(i)}}}\right\rangle \text { (19) }
\end{aligned}
$$

\subsection{Normal intuitionistic generalized hybrid power harmonic operator}

In general, the NIGWPH operator emphasizes the compensation among different input arguments and the importance of overall evaluations, while the NIGOWPH operator stresses the impact of the position of input arguments. In this subsection, based on the 
above two generalized aggregation operators, the Normal Intuitionistic Generalized Hybrid Power Harmonic (NIGHPH) operator is developed to reflect the influence of the overall evaluations and the position of each variable simultaneously.

Definition 13. Let $A_{i}=\left\langle\left(a_{i}, \sigma_{i}\right), u_{i}, v_{i}\right\rangle,(i=$ $1,2, \ldots, n)$ be a collection of NIFNs, and the NIGHPH operator can be defined as follows:

$$
\begin{aligned}
& N \operatorname{IGHPH}\left(A_{1}, A_{2}, \ldots, A_{n}\right) \\
& =\frac{1}{\left(\kappa_{1}\left(\varphi_{\tau(1)}\right)^{\lambda} \oplus \kappa_{2}\left(\varphi_{\tau(2)}\right)^{\lambda} \oplus \cdots \oplus \kappa_{n}\left(\varphi_{\tau(n)}\right)^{\lambda}\right)^{\frac{1}{\lambda}}} \\
& =\frac{1}{\left(\sum_{i=1}^{n} \kappa_{i}\left(\varphi_{\tau(i)}\right)^{\lambda}\right)^{\frac{1}{\lambda}}},
\end{aligned}
$$

where $\lambda \in(-\infty, 0) \cup(0,+\infty), \kappa=\left(\kappa_{1}, \kappa_{2}, \ldots, \kappa_{n}\right)$ are the associated weights, which can be determined as described in Definition 12. $\varphi_{\tau(i)}$ is the $i$-th largest of the power NIFNs $\varphi_{i}=n \frac{1+T\left(A_{i}\right)}{\sum_{i=1}^{n}\left(1+T\left(A_{i}\right)\right)} \frac{1}{A_{i}}(i=1,2, \ldots, n)$, wherein $n$ is the balancing coefficient. Especially, if $\varphi_{\tau(i)}$ is the $i$-th largest of the weighted NIFNs $\varphi_{i}=$ $n w_{i} \frac{1}{A_{i}}(i=1,2, \ldots, n)$ and $w_{i}(i=1,2, \ldots, n)$ is the weight of $A_{i}(i=1,2, \ldots, n)$, then the NIGHPH operator becomes the Normal Intuitionistic Generalized Hybrid Weighted Power Harmonic (NIGHWPH) operator.

Theorem 8. Let $A_{i}=\left\langle\left(a_{i}, \sigma_{i}\right), u_{i}, v_{i}\right\rangle,(i=1,2, \ldots, n)$ be a collection of NIFNs, $\kappa=\left(\kappa_{1}, \kappa_{2}, \ldots, \kappa_{n}\right)$ be the associated weight, and the aggregated value obtained by the NIGHPH operator be:

$$
\begin{array}{rl}
N I G H & P\left(A_{1}, A_{2}, \ldots, A_{n}\right) \\
= & \left\langle\left(\frac{1}{\left(\sum_{i=1}^{n} \kappa_{i} a_{\varphi_{\tau(i)}^{\lambda}}^{\lambda}\right)^{\frac{1}{\lambda}}}, \frac{\sum_{i=1}^{n} \kappa_{i} a_{\varphi_{\tau(i)}^{\lambda-1}}^{n} \sigma_{\varphi_{\tau(i)}}}{\left(\sum_{i=1}^{n} \kappa_{i} a_{\varphi_{\tau(i)}^{\lambda}}^{\lambda}\right)^{\frac{1}{\lambda}+1}}\right)\right. \\
& \left(\frac{\sum_{i=1}^{n}\left|a_{\varphi_{\tau(i)}}\right|^{\lambda} u_{\varphi_{\tau(i)}^{\lambda}}^{\lambda} \kappa_{i}}{\sum_{i=1}^{n}\left|a_{\varphi_{\tau(i)}}\right|^{\lambda} \kappa_{i}}\right)^{\frac{1}{\lambda}}, 1 \\
& -\left(\frac{\sum_{i=1}^{n}\left|a_{\varphi_{\tau(i)}}\right|^{\lambda} \kappa_{i}\left(1-v_{\varphi_{\tau(i)}}\right)^{\lambda}}{\sum_{i=1}^{n}\left|a_{\varphi_{\tau(i)}}\right|^{\lambda} \kappa_{i}}\right\rangle
\end{array}
$$

Similar to the NIGOWPH operator, the NIGHPH operator also satisfies the properties of idempotency and commutativity.

There are some special cases of the NIGHPH operator as follows:

1. If the associated weight of the NIGHPH operator is $\kappa=(1 / n, 1 / n, \ldots, 1 / n)$ and $\lambda=1$, then the
NIGHPH operator becomes a Normal Intuitionistic Power Harmonic (NIPH) operator as:

$$
\begin{array}{rl}
N I P & H\left(A_{1}, A_{2}, \ldots, A_{n}\right)=\frac{1}{\sum_{i=1}^{n} \frac{\left(1+T\left(A_{i}\right)\right)}{\sum_{i=1}^{n}\left(1+T\left(A_{i}\right)\right)} \frac{1}{A_{i}}} \\
& =\left\langle\left(\frac{1}{\sum_{i=1}^{n} \frac{\rho_{i}}{a_{i}}}, \frac{\sum_{i=1}^{n} \frac{\rho_{i} \sigma_{i}}{a_{i}^{2}}}{\left(\sum_{i=1}^{n} \frac{\rho_{i}}{a_{i}}\right)^{2}}\right),\right. \\
& \left.\frac{\sum_{i=1}^{n} \frac{\rho_{i} u_{i}}{\left|a_{i}\right|}}{\sum_{i=1}^{n} \frac{\rho_{i}}{\left|a_{i}\right|}}, \frac{\sum_{i=1}^{n} \frac{\rho_{i} v_{i}}{\left|a_{i}\right|}}{\sum_{i=1}^{n} \frac{\rho_{i}}{\left|a_{i}\right|}}\right\rangle .
\end{array}
$$

2. If $\lambda=1$, then the NIGHPH operator is reduced to the Normal Intuitionistic Hybrid Power Harmonic (NIHPH) operator as follows:

$$
\begin{array}{rl}
N I H & P H\left(A_{1}, A_{2}, \ldots, A_{n}\right)=\frac{1}{\sum_{i=1}^{n} \kappa_{i} \varphi_{\tau(i)}} \\
& =\left\langle\left(\frac{1}{\sum_{i=1}^{n} \kappa_{i} a_{\varphi_{\tau(i)}}}, \frac{\sum_{i=1}^{n} \kappa_{i} \sigma_{\varphi_{\tau(i)}}}{\left(\sum_{i=1}^{n} \kappa_{i} a_{\varphi_{\tau(i)}}\right)^{2}}\right),\right. \\
& \frac{\sum_{i=1}^{n} \kappa_{i}\left|a_{\varphi_{\tau(i)}}\right| u_{\varphi_{\tau(i)}}}{\sum_{i=1}^{n} \kappa_{i}\left|a_{\varphi_{\tau(i)}}\right|}, \\
& \left.\frac{\sum_{i=1}^{n} \kappa_{i}\left|a_{\varphi_{\tau(i)}}\right| v_{\varphi_{\tau(i)}}}{\sum_{i=1}^{n} \kappa_{i}\left|a_{\varphi_{\tau(i)}}\right|}\right\rangle .
\end{array}
$$

3. If $\lambda \rightarrow 0$, then the NIGOWPH operator is reduced to the Normal Intuitionistic Hybrid Geometric Power Harmonic (NIHGPH) operator as follows:

$$
\begin{gathered}
N I H G P\left(A_{1}, A_{2}, \ldots, A_{n}\right)=\frac{1}{\prod_{i=1}^{n}\left(\varphi_{\tau(i)}\right)^{\kappa_{i}}} \\
=\left\langle\left(\frac{1}{\prod_{i=1}^{n} a_{\varphi_{\tau(i)}}^{\kappa_{i}}}, \frac{\sqrt{\sum_{i=1}^{n} \frac{\kappa_{i} \sigma_{\varphi_{\tau(i)}}^{2}}{a_{\varphi_{\tau(i)}}^{2}}}}{\prod_{i=1}^{n} a_{\varphi_{\tau(i)}}^{\kappa_{i}}}\right),\right. \\
\left.\prod_{i=1}^{n} u_{\varphi_{\tau(i)}}^{\kappa_{i}}, 1-\prod_{i=1}^{n}\left(1-v_{\varphi_{\tau(i)}}\right)^{\kappa_{i}}\right\rangle .
\end{gathered}
$$

4. If $\lambda=-1$, then the NIGHPH operator is reduced to the Normal Intuitionistic Hybrid Power (NIHP) operator as:

$$
\begin{array}{r}
\operatorname{NIHP}\left(A_{1}, A_{2}, \ldots, A_{n}\right)=\sum_{i=1}^{n} \frac{\kappa_{i}}{\varphi_{\tau(i)}}= \\
\left\langle\left(\sum_{i=1}^{n} \frac{\kappa_{i}}{a_{\varphi_{\tau(i)}}}, \sum_{i=1}^{n} \frac{\kappa_{i} \sigma_{\varphi_{\tau(i)}}}{a_{\varphi_{\tau(i)}}^{2}}\right),\right. \\
\frac{\sum_{i=1}^{n} \frac{\kappa_{i}}{\left|a_{\varphi_{\tau(i)}}\right|}}{\sum_{i=1}^{n} \frac{\kappa_{i}}{\left|a_{\varphi_{\tau(i)} \mid}\right| u_{\varphi_{\tau(i)}}}}, 1
\end{array}
$$




$$
\left.-\frac{\sum_{i=1}^{n} \frac{\kappa_{i}}{\left|a_{\varphi_{\tau(i)}}\right|}}{\sum_{i=1}^{n} \frac{\kappa_{i}}{\sqrt{a_{\varphi_{\tau(i)}} \mid\left(1-v_{\varphi_{\tau(i)}}\right)}}}\right\rangle .
$$

\section{A MCGDM method under normal intuitionistic fuzzy circumstance}

This section presents the development of MCGDM method in the context of NIFNs. In this method, the proposed normal intuitionistic generalized aggregation operators are used to deal with practical decisionmaking problems.

Concerning MCGDM ranking or selection problem with NIFNs information, we suppose that a group of DMs is denoted by $\left\{d_{1}, d_{2}, \ldots, d_{q}\right\}$ and a set of alternatives is denoted by $\left\{b_{1}, b_{2}, \ldots, b_{n}\right\}$. Each alternative is evaluated under given criteria, denoted by $\left\{c_{1}, c_{2}, \ldots, c_{m}\right\}$. DM $d_{k}$ provides assessments for alternative $b_{i}$ under criteria $c_{j}$ in the form of NIFNs $\phi_{i j}^{k}=$ $\left\langle\left(a_{i j}^{k}, \sigma_{i j}^{k}\right), u_{i j}^{k}, v_{i j}^{k}\right\rangle(i=1,2, \ldots, n ; j=1,2, \ldots, m ; k=$ $1,2, \ldots, q)$. Then, the decision information can be presented as $\phi^{k}=\left(\phi_{i j}^{k}\right)_{m \times n}$.

In practical decision-making problems, the weight information of DMs and criteria are usually unknown because of the uncertainty of decision-making environment and the limitation of DM expertise. In the following, the main procedure of the MCGDM method is presented to handle decision-making problems with completely unknown weight information:

Step 1. Standardize the decision information. To eliminate the influence of different dimensions in the operation process, all evaluation values must be standardized to the same magnitude grade. The normalized evaluation information $\bar{\phi}^{k}=\left(\bar{\phi}_{i j}^{k}\right)_{m \times n}$ can be obtained as follows:

For benefit criteria:

$$
\begin{aligned}
\bar{\phi}_{i j}^{k} & =\left\langle\left(\bar{a}_{i j}^{k}, \bar{\sigma}_{i j}^{k}\right), \bar{u}_{i j}^{k}, \bar{v}_{i j}^{k}\right\rangle \\
& =\left\langle\left(\frac{a_{i j}^{k}}{\max _{i}\left(a_{i j}^{k}\right)}, \frac{\sigma_{i j}^{k}}{\max _{i}\left(\sigma_{i j}^{k}\right)} \frac{\sigma_{i j}^{k}}{a_{i j}^{k}}\right), u_{i j}^{k}, v_{i j}^{k}\right\rangle .
\end{aligned}
$$

For cost criteria:

$$
\begin{aligned}
\bar{\phi}_{i j}^{k} & =\left\langle\left(\bar{a}_{i j}^{k}, \bar{\sigma}_{i j}^{k}\right), \bar{u}_{i j}^{k}, \bar{v}_{i j}^{k}\right\rangle \\
& =\left\langle\left(\frac{\min _{i}\left(a_{i j}^{k}\right)}{a_{i j}^{k}}, \frac{\sigma_{i j}^{k}}{\max _{i}\left(\sigma_{i j}^{k}\right)} \frac{\sigma_{i j}^{k}}{a_{i j}^{k}}\right), u_{i j}^{k}, v_{i j}^{k}\right\rangle .
\end{aligned}
$$

Step 2. Compute the comprehensive support degree. The comprehensive support degree of $\bar{\phi}_{i j}^{k}$ can be calculated as follows:

$$
T\left(\bar{\phi}_{i j}^{k}\right)=\sum_{l=1, l \neq k}^{q} S u p\left(\bar{\phi}_{i j}^{k}, \bar{\phi}_{i j}^{l}\right) \text { and }
$$

$$
\operatorname{Sup}\left(\bar{\phi}_{i j}^{k}, \bar{\phi}_{i j}^{l}\right)=1-\frac{2 d\left(\bar{\phi}_{i j}^{k}, \bar{\phi}_{i j}^{l}\right)}{\sum_{k=1}^{q} \sum_{l=1, l \neq k}^{q} d\left(\bar{\phi}_{i j}^{k}, \bar{\phi}_{i j}^{l}\right)},
$$

where $d\left(\bar{\phi}_{i j}^{k}, \bar{\phi}_{i j}^{l}\right)$ is the Euclidean distance between $\bar{\phi}_{i j}^{k}$ and $\bar{\phi}_{i j}^{l}$, and $\operatorname{Sup}\left(\bar{\phi}_{i j}^{k}, \bar{\phi}_{i j}^{l}\right)$ is the support degree of $\bar{\phi}_{i j}^{k}$ from $\bar{\phi}_{i j}^{l}$, which satisfies the properties described in Definition 9.

Step 3. Aggregate the evaluation of each alternative under individual decision matrix. In the group decision-making problems, the position of evaluations provided by different DMs has no influence on decision results, and the importance of overall evaluations based on all DMs should be highlighted. Thus, to obtain the collective evaluations $\bar{\phi}_{i j}$ of each alternative, the NIGPH operator, which can stress the importance of overall evaluations and capture the weights of input variables, is used to aggregate the individual evaluations of each DM as:

$$
\begin{aligned}
\bar{\phi}_{i j} & =\operatorname{NIGPH}\left(\bar{\phi}_{i j}^{1}, \bar{\phi}_{i j}^{2}, \ldots, \bar{\phi}_{i j}^{q}\right) \\
& =\frac{1}{\left(\sum_{k=1}^{q} \frac{1+T\left(\bar{\phi}_{i j}^{k}\right)}{\sum_{k=1}^{q}\left(1+T\left(\bar{\phi}_{i j}^{k}\right)\right)}\left(\frac{1}{\bar{\phi}_{i j}^{k}}\right)^{\lambda}\right)^{\frac{1}{\lambda}}} .
\end{aligned}
$$

Then, a collective evaluation matrix can be constructed as $O=\left(\bar{\phi}_{i j}\right)$.

Step 4. Compute the associated weight of the NIGHPH operator. First, $\varphi_{i j}=m \frac{1+T\left(\bar{\phi}_{i j}\right)}{\sum_{j=1}^{m}\left(1+T\left(\bar{\phi}_{i j}\right)\right)} \frac{1}{\bar{\phi}_{i j}}$ $\varphi_{i, i n d e x(j)}$ can be calculated based on Step 3. Second, the $j$-th largest value can be obtained by comparing $\varphi_{i j}$. Moreover, the support degree and the comprehensive support degree can be computed as follows:

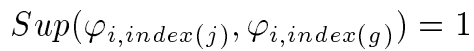

$$
\begin{aligned}
& -\frac{2 d\left(\varphi_{i, i n \operatorname{dex}(j)}, \varphi_{i, i n \operatorname{dex}(g)}\right)}{\sum_{j=1}^{m} \sum_{g=1, g \neq j}^{m} d\left(\varphi_{i, i n \operatorname{dex}(j)}, \varphi_{i, i n \operatorname{dex}(g)}\right)}, \text { and } \\
& T\left(\varphi_{i, \text { index }(j)}\right)=\sum_{g=1, g \neq j}^{m} \operatorname{Sup}\left(\varphi_{i, \operatorname{index}(j)}, \varphi_{i, \operatorname{index}(g)}\right) .
\end{aligned}
$$

The BUM function is considered as $g(x)=x$ and, then, $\omega_{j}=\frac{1+T\left(\varphi_{i, i n \operatorname{dex}(j)}\right)}{\sum_{j=1}^{m}\left(1+T\left(\varphi_{i, \text { index }(j)}\right)\right)}$.

Step 5. Aggregate the evaluations of each alternative under all criteria. Evaluations may vary significantly after the first aggregation. To acquire valid results, the evaluations should be preprocessed before the next aggregation. For example, the rearrangement of all variables is an effective mean. Therefore, to obtain the comprehensive evaluation $\phi_{i}$ of each alternative, the NIGHPH operator, which can consider the position of input variables and the influence of overall 
evaluations simultaneously, is used to integrate the collective evaluations under all criteria as follows:

$$
\begin{aligned}
\phi_{i} & =\operatorname{NIGHPH}\left(\bar{\phi}_{i 1}, \bar{\phi}_{i 2}, \ldots, \bar{\phi}_{i m}\right) \\
& =\frac{1}{\left(\sum_{j=1}^{m} \omega_{j}\left(\varphi_{i, i n \operatorname{dex}(j)}\right)^{\lambda}\right)^{\frac{1}{\lambda}}} .
\end{aligned}
$$

Step 6. Calculate the scoring value of each alternative. The scoring value $S\left(\phi_{i}\right)$ can be calculated using Eq. (6) according to Definition 8.

Step 7. Rank all alternatives and select the best one. The order of all alternatives can be ranked and the best alternative can be identified according to the scoring value $S\left(\phi_{i}\right)$.

In some cases, if the weight information of the MCGDM problems can be acquired by effective methods, then a method can also be developed to address such decision-making problems. The steps of this method are slightly different from the above method. The distinct steps are described in the following.

In Step 2, the comprehensive weighted support degree must be computed as:

$$
T\left(\bar{\phi}_{i j}^{k}\right)=\sum_{l=1, l \neq k}^{q} \zeta_{l} S u p\left(\bar{\phi}_{i j}^{k}, \bar{\phi}_{i j}^{l}\right),
$$

where $\zeta=\left(\zeta_{1}, \zeta_{2}, \ldots, \zeta_{q}\right)$ is the weight vector of DMs.

In Step 3, the NIGWPH operator must be used to aggregate the individual evaluations of each DM as follows:

$$
\begin{aligned}
\bar{\phi}_{i j} & =N I G W P H\left(\bar{\phi}_{i j}^{1}, \bar{\phi}_{i j}^{2}, \ldots, \bar{\phi}_{i j}^{q}\right) \\
& =\frac{1}{\left(\sum_{k=1}^{q} \frac{\zeta_{k}\left(1+T\left(\bar{\phi}_{i j}^{k}\right)\right)}{\sum_{k=1}^{q} \zeta_{k}\left(1+T\left(\bar{\phi}_{i j}^{k}\right)\right)}\left(\frac{1}{\bar{\phi}_{i j}^{k}}\right)^{\lambda}\right)^{\frac{1}{\lambda}}} .
\end{aligned}
$$

In Step 5, the NIGHWPH operator must be used to aggregate the collective evaluations $\bar{\phi}_{i j}$, obtained in Step 3, as follows:

$$
\begin{aligned}
\phi_{i} & =\operatorname{NIGHWPH}\left(\bar{\phi}_{i 1}, \bar{\phi}_{i 2}, \ldots, \bar{\phi}_{i m}\right) \\
& =\frac{1}{\left(\sum_{j=1}^{m} \omega_{j}\left(\varphi_{i, i n \operatorname{dex}(j)}\right)^{\lambda}\right)^{\frac{1}{\lambda}}}
\end{aligned}
$$

where $\varphi_{i, i n d e x(j)}$ is the $j$-th largest of the weighted NIFNs $\varphi_{i j}=n \delta_{j} \frac{1}{\bar{\phi}_{i j}}$, and $\delta=\left(\delta_{1}, \delta_{2}, \ldots, \delta_{m}\right)$ is the weight vector of criteria.

\section{Illustrative example}

This section provides a practical decision-making problem to highlight the applicability of the proposed method. Furthermore, the availability and effectiveness of the proposed method are confirmed through the sensitivity analysis and the comparative analysis with existing methods.

A Mining Bureau evaluates the safety conditions of four coal mines in an area according to correlative assessment methods and production regulations. The four coal mines are denoted by $\left\{b_{1}, b_{2}, b_{3}, b_{4}\right\}$. To effectively assess these coal mines and select the safest one, a professional team including three DMs $\left\{d_{1}, d_{2}, d_{3}\right\}$ is formed. Many factors affect the safety environment, and the following four criteria are considered based on detailed investigation: $c_{1}$ technological equipment; $c_{2}$, geological conditions; $c_{3}$, human diathesis; and $c_{4}$, management quality [60]. The evaluation values of alternative $b_{i}$ under criteria $c_{j}$ are provided by DMs $d_{l}$ in the form of NIFNs $\phi_{i j}^{k}=\left\langle\left(a_{i j}^{k}, \sigma_{i j}^{k}\right), u_{i j}^{k}, v_{i j}^{k}\right\rangle$, $(i=1,2,3 ; j=1,2,3 ; k=1,2,3)$, and the normal intuitionistic fuzzy evaluation information $\phi^{k}=\left(\phi_{i j}^{k}\right)_{4 \times 4}$ is shown in Tables 1-3.

Table 1. Evaluations of Normal Intuitionistic Fuzzy Numbers (NIFNs) provided by $d_{1}$.

\begin{tabular}{ccccc}
\hline & $\boldsymbol{c}_{\boldsymbol{1}}$ & $\boldsymbol{c}_{\boldsymbol{2}}$ & $\boldsymbol{c}_{\boldsymbol{3}}$ & $\boldsymbol{c}_{\boldsymbol{4}}$ \\
\hline$b_{1}$ & $\langle(5.5,0.4), 0.7,0.3\rangle$ & $\langle(8,0.35), 0.5,0.4\rangle$ & $\langle(6,0.4), 0.8,0.1\rangle$ & $\langle(5,0.35), 0.6,0.4\rangle$ \\
$b_{2}$ & $\langle(7,0.3), 0.6,0.4\rangle$ & $\langle(5.5,0.6), 0.75,0.2\rangle$ & $\langle(5,0.3), 0.7,0.3\rangle$ & $\langle(7,0.5), 0.8,0.1\rangle$ \\
$b_{3}$ & $\langle(5,0.5), 0.6,0.35\rangle$ & $\langle(4,0.3), 0.6,0.3\rangle$ & $\langle(7.5,0.5), 0.5,0.4\rangle$ & $\langle(6,0.6), 0.7,0.3\rangle$ \\
$b_{4}$ & $\langle(8,0.6), 0.65,0.3\rangle$ & $\langle(6,0.4), 0.8,0.1\rangle$ & $\langle(7,0.4), 0.6,0.4\rangle$ & $\langle(7.5,0.4), 0.65,0.3\rangle$ \\
\hline
\end{tabular}

Table 2. Evaluations of Normal Intuitionistic Fuzzy Numbers (NIFNs) provided by $d_{2}$.

\begin{tabular}{ccccc}
\hline & $\boldsymbol{c}_{\boldsymbol{1}}$ & $\boldsymbol{c}_{\boldsymbol{2}}$ & $\boldsymbol{c}_{\boldsymbol{3}}$ & $\boldsymbol{c}_{\boldsymbol{4}}$ \\
\hline$b_{1}$ & $\langle(7,0.6), 0.6,0.4\rangle$ & $\langle(8,0.4), 0.8,0.2\rangle$ & $\langle(6,0.2), 0.6,0.4\rangle$ & $\langle(7,0.6), 0.65,0.3\rangle$ \\
$b_{2}$ & $\langle(5,0.3), 0.7,0.3\rangle$ & $\langle(6,0.2), 0.6,0.3\rangle$ & $\langle(3.5,0.3), 0.6,0.4\rangle$ & $\langle(5,0.3), 0.7,0.2\rangle$ \\
$b_{3}$ & $\langle(7,0.2), 0.7,0.2\rangle$ & $\langle(7,0.5), 0.6,0.35\rangle$ & $\langle(5,0.5), 0.8,0.2\rangle$ & $\langle(6,0.4), 0.6,0.25\rangle$ \\
$b_{4}$ & $\langle(4,0.5), 0.8,0.1\rangle$ & $\langle(6.5,0.5), 0.6,0.4\rangle$ & $\langle(6,0.4), 0.7,0.3\rangle$ & $\langle(8,0.6), 0.5,0.5\rangle$ \\
\hline
\end{tabular}


Table 3. Evaluations of Normal Intuitionistic Fuzzy Numbers (NIFNs) provided by $d_{3}$.

\begin{tabular}{ccccc}
\hline & $\boldsymbol{c}_{\boldsymbol{1}}$ & $\boldsymbol{c}_{\boldsymbol{2}}$ & $\boldsymbol{c}_{\boldsymbol{3}}$ & $\boldsymbol{c}_{\boldsymbol{4}}$ \\
\hline$b_{1}$ & $\langle(6,0.5), 0.7,0.3\rangle$ & $\langle(7.5,0.5), 0.75,0.2\rangle$ & $\langle(7,0.5), 0.7,0.3\rangle$ & $\langle(4,0.2), 0.6,0.4\rangle$ \\
$b_{2}$ & $\langle(5,0.2), 0.65,0.35\rangle$ & $\langle(8,0.4), 0.7,0.3\rangle$ & $\langle(4,0.3), 0.8,0.2\rangle$ & $\langle(7,0.5), 0.8,0.2\rangle$ \\
$b_{3}$ & $\langle(7,0.4), 0.8,0.2\rangle$ & $\langle(6.5,0.35), 0.6,0.4\rangle$ & $\langle(6,0.4), 0.6,0.35\rangle$ & $\langle(7.5,0.6), 0.65,0.35\rangle$ \\
$b_{4}$ & $\langle(7.5,0.6), 0.5,0.45\rangle$ & $\langle(6,0.6), 0.5,0.5\rangle$ & $\langle(5.5,0.5), 0.7,0.3\rangle$ & $\langle(6.5,0.5), 0.65,0.3\rangle$ \\
\hline
\end{tabular}

Table 4. Normalized evaluations of $d_{1}$.

\begin{tabular}{ccccc}
\hline & \multicolumn{1}{c}{$\boldsymbol{c}_{\boldsymbol{1}}$} & $\boldsymbol{c}_{\boldsymbol{2}}$ & $\boldsymbol{c}_{\boldsymbol{3}}$ & $\boldsymbol{c}_{\boldsymbol{4}}$ \\
\hline$b_{1}$ & $\langle(0.69,0.05), 0.7,0.3\rangle$ & $\langle(1,0.03), 0.5,0.4\rangle$ & $\langle(0.8,0.05), 0.8,0.1\rangle$ & $\langle(0.67,0.04), 0.6,0.4\rangle$ \\
$b_{2}$ & $\langle(0.88,0.02), 0.6,0.4\rangle$ & $\langle(0.69,0.11), 0.75,0.2\rangle$ & $\langle(0.67,0.04), 0.7,0.3\rangle$ & $\langle(0.93,0.06), 0.8,0.1\rangle$ \\
$b_{3}$ & $\langle(0.63,0.08), 0.6,0.35\rangle$ & $\langle(0.5,0.04), 0.6,0.3\rangle$ & $\langle(1,0.07), 0.5,0.4\rangle$ & $\langle(0.8,0.1), 0.7,0.3\rangle$ \\
$b_{4}$ & $\langle(1,0.08), 0.65,0.3\rangle$ & $\langle(0.75,0.04), 0.8,0.1\rangle$ & $\langle(0.93,0.05), 0.6,0.4\rangle$ & $\langle(1,0.04), 0.65,0.3\rangle$ \\
\hline
\end{tabular}

Table 5. Normalized evaluations of $d_{2}$.

\begin{tabular}{ccccc}
\hline & $\boldsymbol{c}_{\boldsymbol{1}}$ & $\boldsymbol{c}_{\boldsymbol{2}}$ & $\boldsymbol{c}_{\boldsymbol{3}}$ & $\boldsymbol{c}_{\boldsymbol{4}}$ \\
\hline$b_{1}$ & $\langle(1,0.09), 0.6,0.4\rangle$ & $\langle(1,0.04), 0.8,0.2\rangle$ & $\langle(1,0.01), 0.6,0.4\rangle$ & $\langle(0.88,0.09), 0.65,0.3\rangle$ \\
$b_{2}$ & $\langle(0.71,0.03), 0.7,0.3\rangle$ & $\langle(0.75,0.01), 0.6,0.3\rangle$ & $\langle(0.58,0.05), 0.6,0.4\rangle$ & $\langle(0.63,0.03), 0.7,0.2\rangle$ \\
$b_{3}$ & $\langle(1,0.01), 0.7,0.2\rangle$ & $\langle(0.88,0.07), 0.6,0.35\rangle$ & $\langle(0.83,0.1), 0.8,0.2\rangle$ & $\langle(0.75,0.04), 0.6,0.25\rangle$ \\
$b_{4}$ & $\langle(0.57,0.10), 0.8,0.1\rangle$ & $\langle(0.81,0.08), 0.6,0.4\rangle$ & $\langle(1,0.05), 0.7,0.3\rangle$ & $\langle(1,0.08), 0.5,0.5\rangle$ \\
\hline
\end{tabular}

Table 6. Normalized evaluations of $d_{3}$.

\begin{tabular}{ccccc}
\hline & $\boldsymbol{c}_{\boldsymbol{1}}$ & $\boldsymbol{c}_{\boldsymbol{2}}$ & $\boldsymbol{c}_{\boldsymbol{3}}$ & $\boldsymbol{c}_{\boldsymbol{4}}$ \\
\hline$b_{1}$ & $\langle(0.8,0.07), 0.7,0.3\rangle$ & $\langle(0.94,0.06), 0.75,0.2\rangle$ & $\langle(1,0.07), 0.7,0.3\rangle$ & $\langle(0.53,0.02), 0.6,0.4\rangle$ \\
$b_{2}$ & $\langle(0.67,0.01), 0.65,0.35\rangle$ & $\langle(1,0.03), 0.7,0.3\rangle$ & $\langle(0.57,0.05), 0.8,0.2\rangle$ & $\langle(0.93,0.06), 0.8,0.2\rangle$ \\
$b_{3}$ & $\langle(0.93,0.04), 0.8,0.2\rangle$ & $\langle(0.81,0.03), 0.6,0.4\rangle$ & $\langle(0.86,0.05), 0.6,0.35\rangle$ & $\langle(1,0.08), 0.65,0.35\rangle$ \\
$b_{4}$ & $\langle(1,0.08), 0.5,0.45\rangle$ & $\langle(0.75,0.1), 0.5,0.5\rangle$ & $\langle(0.79,0.09), 0.7,0.3\rangle$ & $\langle(0.87,0.06), 0.65,0.3\rangle$ \\
\hline
\end{tabular}

\subsection{Illustration of the proposed method}

The main procedures for evaluating the safest coal mine are presented in the following:

Step 1. Standardize the decision information. Considering that all the criteria are of benefit type, the normalized evaluations are shown in Tables 4-6.

Step 2. Compute the comprehensive support degree. First, the distance between $\bar{\phi}_{i j}^{k}$ and $\bar{\phi}_{i j}^{l}$ should be computed according to Definition 4. For example, $d\left(\bar{\phi}_{11}^{1}, \bar{\phi}_{11}^{2}\right)=0.1194, d\left(\bar{\phi}_{11}^{1}, \bar{\phi}_{11}^{3}\right)=0.0794$, and $d\left(\bar{\phi}_{11}^{2}, \bar{\phi}_{11}^{3}\right)=0.0401$. Second, the support degree can be calculated as $\operatorname{Sup}\left(\bar{\phi}_{11}^{1}, \bar{\phi}_{11}^{2}\right)=0.5002$, $\operatorname{Sup}\left(\bar{\phi}_{11}^{1}, \bar{\phi}_{11}^{3}\right)=0.6675$, and $\operatorname{Sup}\left(\bar{\phi}_{11}^{2}, \bar{\phi}_{11}^{3}\right)=0.8323$. Then, the comprehensive support degree can be obtained as $T\left(\bar{\phi}_{11}^{1}\right)=1.1677, T\left(\bar{\phi}_{11}^{2}\right)=1.3325$, and $T\left(\bar{\phi}_{11}^{3}\right)=1.4998$. All comprehensive support degrees are shown in the following three matrices.

$$
\begin{aligned}
T\left(\bar{\phi}_{i j}^{1}\right) & =\left[\begin{array}{cccc}
1.1677 & 1.1471 & 1.4995 & 1.5 \\
1.3642 & 1.4669 & 1.454 & 1.4282 \\
1.0229 & 1.139 & 1.4999 & 1.4881 \\
1.1306 & 1.2142 & 1.4741 & 1.2422
\end{array}\right], \\
T\left(\bar{\phi}_{i j}^{2}\right) & =\left[\begin{array}{cccc}
1.3325 & 1.3541 & 1.0971 & 1.1479 \\
1.4968 & 1.379 & 1.0517 & 1.0718 \\
1.4855 & 1.3636 & 1.0536 & 1.3068 \\
1.3824 & 1.5 & 1.0862 & 1.259
\end{array}\right], \\
T\left(\bar{\phi}_{i j}^{3}\right) & =\left[\begin{array}{cccc}
1.4998 & 1.4987 & 1.4034 & 1.3521 \\
1.139 & 1.1541 & 1.4943 & 1.5 \\
1.4915 & 1.4974 & 1.4465 & 1.205 \\
1.487 & 1.2858 & 1.4397 & 1.4988
\end{array}\right] .
\end{aligned}
$$

Step 3. Aggregate the evaluations of each alternative under individual decision matrix. Let $\lambda=1$ and then, 
Table 7. Collective evaluations.

\begin{tabular}{ccccc}
\hline & $\boldsymbol{c}_{\boldsymbol{1}}$ & $\boldsymbol{c}_{\boldsymbol{2}}$ & $\boldsymbol{c}_{\boldsymbol{3}}$ & $\boldsymbol{c}_{\boldsymbol{4}}$ \\
\hline$b_{1}$ & $\langle(0.81,0.07), 0.67,0.33\rangle$ & $\langle(0.98,0.04), 0.69,0.26\rangle$ & $\langle(0.92,0.05), 0.71,0.25\rangle$ & $\langle(0.66,0.04), 0.61,0.38\rangle$ \\
$b_{2}$ & $\langle(0.74,0.02), 0.65,0.35\rangle$ & $\langle(0.79,0.06), 0.68,0.26\rangle$ & $\langle(0.61,0.04), 0.71,0.29\rangle$ & $\langle(0.81,0.05), 0.76,0.17\rangle$ \\
$b_{3}$ & $\langle(0.83,0.06), 0.69,0.26\rangle$ & $\langle(0.70,0.05), 0.60,0.34\rangle$ & $\langle(0.90,0.07), 0.63,0.32\rangle$ & $\langle(0.83,0.07), 0.65,0.29\rangle$ \\
$b_{4}$ & $\langle(0.80,0.10), 0.68,0.25\rangle$ & $\langle(0.77,0.07), 0.63,0.34\rangle$ & $\langle(0.89,0.07), 0.67,0.33\rangle$ & $\langle(0.95,0.06), 0.60,0.36\rangle$ \\
\hline
\end{tabular}

Table 8. Rankings with the varying values of $\lambda$.

\begin{tabular}{|c|c|c|c|c|c|}
\hline \multirow[b]{2}{*}{$\lambda$} & \multicolumn{4}{|c|}{ Scoring value of each alternatives } & \multirow[b]{2}{*}{ Ranking } \\
\hline & $S\left(\phi_{1}\right)$ & $S\left(\phi_{2}\right)$ & $S\left(\phi_{3}\right)$ & $S\left(\phi_{4}\right)$ & \\
\hline$\lambda=-10$ & 0.2915 & 0.2965 & 0.3365 & 0.2489 & $b_{4} \succ b_{1} \succ b_{2} \succ b_{3}$ \\
\hline$\lambda=-8$ & 0.2923 & 0.2996 & 0.3312 & 0.2236 & $b_{4} \succ b_{1} \succ b_{2} \succ b_{3}$ \\
\hline$\lambda=-6$ & 0.2968 & 0.3086 & 0.3255 & 0.2185 & $b_{4} \succ b_{1} \succ b_{2} \succ b_{3}$ \\
\hline$\lambda=-2$ & 0.2896 & 0.3195 & 0.3178 & 0.2912 & $b_{1} \succ b_{4} \succ b_{3} \succ b_{2}$ \\
\hline$\lambda=-1$ & 0.2863 & 0.3297 & 0.3166 & 0.2965 & $b_{1} \succ b_{4} \succ b_{3} \succ b_{2}$ \\
\hline$\lambda \rightarrow 0$ & 0.2971 & 0.3362 & 0.3249 & 0.3152 & $b_{1} \succ b_{4} \succ b_{3} \succ b_{2}$ \\
\hline$\lambda=1$ & 0.2951 & 0.3352 & 0.3224 & 0.2957 & $b_{1} \succ b_{4} \succ b_{3} \succ b_{2}$ \\
\hline$\lambda=2$ & 0.2922 & 0.3296 & 0.3192 & 0.2724 & $b_{4} \succ b_{1} \succ b_{3} \succ b_{2}$ \\
\hline$\lambda=6$ & 0.2806 & 0.2795 & 0.3209 & 0.1818 & $b_{4} \succ b_{2} \succ b_{1} \succ b_{3}$ \\
\hline$\lambda=8$ & 0.2881 & 0.2646 & 0.3362 & 0.1651 & $b_{4} \succ b_{2} \succ b_{1} \succ b_{3}$ \\
\hline$\lambda=10$ & 0.2992 & 0.2572 & 0.3520 & 0.1553 & $b_{4} \succ b_{2} \succ b_{1} \succ b_{3}$ \\
\hline
\end{tabular}

the individual evaluations can be aggregated using the NIGPH operator, and the collective evaluations can be obtained, as shown in Table 7 .

Step 4. Compute the associated weight of the NIGHPH operator. According to the obtained collective evaluations, $\varphi_{i j}=m \frac{1+T\left(\bar{\phi}_{i j}\right)}{\sum_{j=1}^{m}\left(1+T\left(\bar{\phi}_{i j}\right)\right)} \frac{1}{\bar{\phi}_{i j}}$ can be calculated and ranked. Moreover, after computing the support degree and the comprehensive support degree, the associated weight of the NIGHPH operator under each criterion can be computed as follows:

$$
\omega_{i j}=\left[\begin{array}{cccc}
0.2581 & 0.2351 & 0.2587 & 0.2481 \\
0.255 & 0.2604 & 0.2234 & 0.2612 \\
0.244 & 0.2573 & 0.2416 & 0.2571 \\
0.2332 & 0.2611 & 0.2611 & 0.2446
\end{array}\right] .
$$

Step 5. Aggregate the evaluations of each alternative under all criteria. Utilizing the NIGHPH operator to aggregate the collective evaluations $\bar{\phi}_{i j}$ obtained in Step 3, the comprehensive evaluations of each alternative can be obtained as:

$$
\begin{aligned}
& \phi_{1}=\langle(0.8255,0.0493), 0.6683,0.3088\rangle, \\
& \phi_{2}=\langle(0.7331,0.0438), 0.7001,0.2704\rangle, \\
& \phi_{3}=\langle(0.8118,0.0616), 0.6428,0.3045\rangle, \\
& \phi_{4}=\langle(0.8496,0.0778), 0.6462,0.3186\rangle .
\end{aligned}
$$

Step 6. Calculate the scoring value of each alternative. The scoring value $S\left(\phi_{1}\right)$ of the comprehensive evaluation $\phi_{i}$ of each alternative can be calculated by using Eq. (6) as:

$$
\begin{aligned}
& S\left(\phi_{1}\right)=0.2951, \quad S\left(\phi_{2}\right)=0.3352, \\
& S\left(\phi_{3}\right)=0.3224, \quad \text { and } \quad S\left(\phi_{4}\right)=0.2957 .
\end{aligned}
$$

Step 7. Rank all alternatives and select the best one. From Step 6, it can be seen that the final ranking of the four coal mines is $b_{1} \succ b_{4} \succ b_{3} \succ b_{2}$. Thus, the safest coal mine is identified as $b_{1}$.

\subsection{Sensitivity analysis}

The above ranking of safe coal mines is obtained by using the NIGPH and NIGHPH operators with $\lambda=1$. The study should discuss whether or not the final ranking changes when different values of parameter $\lambda$, which is involved in the normal intuitionistic generalized aggregation operators, are used.

The given example can also be used to conduct the sensitivity analysis. The results obtained based on different values of parameter $\lambda$ are shown in Table 8 . In addition, the scoring values of alternatives obtained using the varying values of $\lambda$ are shown in Figure 2 .

The above data shows that the rankings obtained based on the normal intuitionistic generalized aggregation operators apparently change with the varying 


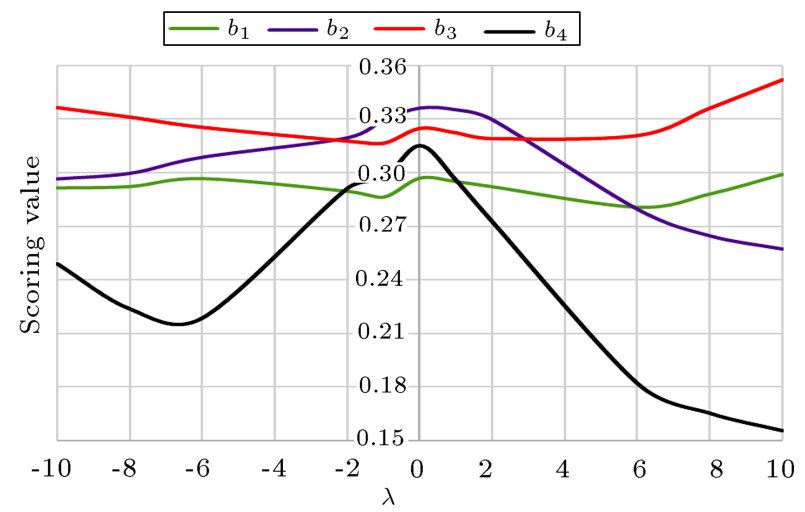

Figure 2. Scoring values of alternatives with the varying values of $\lambda$.

values of $\lambda$ : when $-10 \leq \lambda<-2$, the ranking is $b_{4} \succ b_{1} \succ b_{2} \succ b_{3}$; when $-2 \leq \lambda \leq 1$, the ranking is $b_{1} \succ b_{4} \succ b_{3} \succ b_{2}$; when $1<\lambda \leq 3$, the ranking is $b_{4} \succ b_{1} \succ b_{3} \succ b_{2}$; when $3<\lambda<6$, the ranking is $b_{4} \succ b_{1} \succ b_{2} \succ b_{3}$; and when $\lambda \geq 6$, the ranking is $b_{4} \succ b_{2} \succ b_{1} \succ b_{3}$. This finding indicates that normal intuitionistic generalized aggregation operators are sensitive to varying values of $\lambda$ under the situation in the above coal mine safety evaluation problem.

The above discussion demonstrates that the values of $\lambda$ can influence the final ranking of the safe coal mines. In general, $\lambda$ is correlated with the thinking mode of the DM. The bigger the values of $\lambda$, the more optimistic the DM is; meanwhile, the smaller the values of $\lambda$, the more pessimistic the DM is. Moreover, $\lambda<0$ indicates that the DM is negative, and $\lambda>0$ indicates that the DM is positive. Therefore, the proposed method for evaluating coal mine safety is very flexible, and DMs can choose appropriate values of $\lambda$ according to their preferences and actual situations to obtain the most precise result.

\subsection{Comparison analysis and discussion}

To further verify the feasibility and validity of the proposed method under normal intuitionistic fuzzy environment, the comparison analysis for two existing methods is conducted.
1. Comparison analysis for the existing methods using NIFNs. Wang et al. [22] proposed the MCDM method based on NIFNs by developing a series of aggregation operators, such as Normal Intuitionistic Fuzzy-Induced Ordered Weighted Averaging (NIFIOWA) operator, Normal Intuitionistic Fuzzy-Induced Ordered Weighted Geometric Averaging (NIFIOWGA) operator, and (NIFIGOWA) operator. Moreover, a practical MCDM problem was handled using the NIFIGOWA operator in [22]. The obtained rankings with varying values of $\lambda$ are shown in Table 9. To ensure an effective comparison, the NIGIOWPH operator proposed in Section 4 is used to address the MCDM problem in [22]. The following procedures need to be provided.

First, the comprehensive evaluation of each alternative can be calculated using the NIGIOWPH operator based on the normalized decision matrix. Second, the scoring value of each alternative can be calculated using Eq. (6) and the ranking can be determined. Finally, the rankings obtained by the NIGIOWPH operator with varying values of $\lambda$ are shown in Table 9. For example, when $\lambda=1$, the scoring values of alternatives are obtained as $S\left(\phi_{1}\right)=0.3783, S\left(\phi_{2}\right)=0.2698, S\left(\phi_{3}\right)=0.4000$, and $S\left(\phi_{4}\right)=0.2211$; and the ranking of alternatives is therefore determined as $b_{4} \succ b_{2} \succ b_{1} \succ b_{3}$.

The data in Table 9 shows that the rankings obtained by the proposed approach and the method in [22] are slightly distinct with the varying values of $\lambda$. The reasons for the inconsistency of rankings are explained in the following.

First, although both the NIFIGOWA operator in [22] and our proposed NIGIOWPH operator are based on IOWA, NIGIOWPH has better capability to deal with fuzzy information fusion than NIFIGOWA. By combining PA and HM, NIGIOWPH allows aggregated values to support each other with consideration of the interrelationships among them and can relive the impact of exceptional data in the aggregation process. Second, the method in [22] provided the weight information

Table 9. Rankings using different methods with the varying values of $\lambda$.

\begin{tabular}{lll}
\hline$\lambda$ & Method with the NIFIGOWA operator & Method with the NIGIOWPH operator \\
\hline$\lambda \rightarrow 0$ & $b_{4} \succ b_{2} \succ b_{1} \succ b_{3}$ & $b_{4} \succ b_{2} \succ b_{1} \succ b_{3}$ \\
$\lambda=1$ & $b_{4} \succ b_{2} \succ b_{1} \succ b_{3}$ & $b_{4} \succ b_{2} \succ b_{1} \succ b_{3}$ \\
$\lambda=2$ & $b_{4} \succ b_{2} \succ b_{3} \succ b_{1}$ & $b_{4} \succ b_{2} \succ b_{1} \succ b_{3}$ \\
$\lambda=5$ & $b_{2} \succ b_{4} \succ b_{3} \succ b_{1}$ & $b_{4} \succ b_{2} \succ b_{1} \succ b_{3}$ \\
$\lambda=10$ & $b_{2} \succ b_{4} \succ b_{3} \succ b_{1}$ & $b_{4} \succ b_{2} \succ b_{3} \succ b_{1}$ \\
$\lambda=20$ & $b_{2} \succ b_{4} \succ b_{3} \succ b_{1}$ & $b_{4} \succ b_{2} \succ b_{3} \succ b_{1}$ \\
$\lambda=50$ & $b_{2} \succ b_{4} \succ b_{3} \succ b_{1}$ & $b_{4} \succ b_{2} \succ b_{3} \succ b_{1}$ \\
\hline
\end{tabular}


subjectively, which would affect the precision of the final results, whereas our proposed method could effectively judge and weigh the importance of arguments and appropriately assign weights to them in the aggregation process by using the PA operator. Finally, the comparison approaches of NIFNs in [22] and our proposed method are significantly distinct. As discussed in Section 3, the comparison approach of NIFNs in [22] has many non-negligible problems, which may lead to unreasonable ranking results. To address the drawbacks of the existing comparison approach, this study developed an effective normal intuitionistic fuzzy ranking method by integrating the expectation and variance of normal distribution with the hesitation degree and distance measure of NIFNs.

In addition, the above comparison analysis illustrates that the proposed method can not only solve MCGDM problems, but also address MCDM problems effectively.

2. Comparison analysis for the existing method using TIFNs. Wan et al. [14] proposed MCGDM method under the triangular intuitionistic fuzzy environment. This method developed an extended VIKOR model to balance the maximum of utility for the majority and the minimum of the individual regret for the opponent. Now, the preceding example is assessed using the method in [14].

First, the NIFNs should be transformed into TIFNs according to the procedures in [22]. For example, let $A=\langle(a, \sigma), u, v\rangle$ be an NIFN and then, the transformed TIFN can be obtained as $\tilde{A}=\langle(\tilde{a}, \tilde{b}, \tilde{c}), \tilde{u}, \tilde{v}\rangle$, where $\tilde{a}=a-3 \sigma, \tilde{b}=a$, $\tilde{c}=a+3 \sigma, \tilde{u}=u$, and $\tilde{v}=v$.

Second, the weighted possibility means can be determined through the defuzzification method, and the individual weights of criteria given by the three DMs can be calculated through the Shannon entropy theory. Then, the DMs weights and criteria weights can be computed as $D W=(0.3325,0.3247,0.3428)$ and $C W=$ $(0.2088,0.3168,0.2095,0.2649)$, respectively.

Third, the individual evaluations are aggregated by utilizing the TIF-WA operator, and the group decision matrix can be constructed. Then, the group utility values can be computed as $G\left(b_{1}\right)=$ $0.4434, G\left(b_{2}\right)=0.3390, G\left(b_{3}\right)=0.4634$, and $G\left(b_{4}\right)=0.4966$; and the individual regret values can be computed as $I\left(b_{1}\right)=0.1564, I\left(b_{2}\right)=0.1127$, $I\left(b_{3}\right)=0.1821$, and $I\left(b_{4}\right)=0.1983$.

Finally, the ranking is identified as $b_{2} \succ b_{1} \succ$ $b_{3} \succ b_{4}$ according to the closeness coefficient of alternatives.

Based on the discussion above, it can be seen that the ranking of alternatives obtained by the extended VIKOR model is quite different from that obtained by the developed method in this paper. There are some possible reasons for this difference in rankings.

First, there is an essential distinction between the ideas of the two methods. The method of Wan et al. [14] develops an extended VIKOR model to deal with MCGDM problems and mainly focuses on the measurement among different evaluations, while the proposed approach uses a series of NIPH aggregation operators to address MCGDM problems, emphasizing the significance of comprehensive evaluations. Second, the way of determining the final ranking is different between the two methods. The method of Wan et al. [14] employs the group utility values and individual regret values to determine the closeness coefficient of alternatives and obtain the ranking, while the proposed method presents an effective ranking method to decide the final ranking, which considers all variables in NIFNs and the distance to the positive ideal solution comprehensively. Third, the proposed method utilizes the PA operator to appropriately assign weights to the input variables in the aggregation process according to the support measure and importance of these variables, whereas the method of Wan et al. [14] uses some auxiliary tools, such as Shannon entropy, evidence theory, Bayes approximation to determine the weights of criteria and DMs.

In addition, there are some non-negligible shortcomings about the method of Wan et al. [14]. First, the normalization of evaluation information is usually conducted in the initial stage, however, the method of Wan et al. [14] normalizes the collective decision information obtained by aggregating the individual evaluations. This strategy amplifies the negative impact of certain exception variables to some extent. Second, as discussed in [42], the operations of TIFNs in [14] have some obvious defects, which will affect the precision of the final result. Third, to determine the weights of criteria and DMs, the triangular intuitionistic fuzzy evaluation information needs to be transformed into crisp weighted possibility means, and many complicated procedures are used in [14]. The weight determination method in [14] is tedious and complicated, and the transformation from TIFNs to crisp values will lose and distort the original information. Finally, the method of Wan et al. [14] involves many complicated steps, which increase the calculation complexity. Although the proposed approach based on aggregation operators also needs a large number of calculations, the operations among all steps are regular and correlative. In this way, the computation efficiency of the proposed approach can be easily improved using such tools as MATLAB. 


\section{Conclusions}

Normal Intuitionistic Fuzzy Number (NIFN) is greatly effective in presenting uncertain information with normal distribution which can reflect many social and natural phenomena; thereby, the research of MultiCriteria Group Decision-Making (MCGDM) method with NIFNs is of paramount significance. A reliable ranking method for NIFNs was introduced after reviewing the defects in the existing comparison method of NIFNs, Furthermore, inspired by the Power Average (PA) and Harmonic Mean (HM) operators, three normal intuitionistic generalized aggregation operators were proposed. In addition, the MCGDM approach under normal intuitionistic fuzzy environment was developed. Finally, this method was tested by a practical coal mine safety evaluation problem, and it was further validated through sensitivity analysis. The comparison results demonstrated that the proposed method could provide more reliable and precise outcome than other methods. Therefore, the proposed method has great application potential in solving MCGDM or MultiCriteria Decision-Making (MCDM) problems.

The main contribution of this study is the integration of NIFNs with the PA and HM operators. In this way, the PA operator can judge the importance of the input variables and assign weights to these variables in the aggregation process effectively, and the HM operator can pay greater attention to the central tendency data. Moreover, the proposed method can address both MCGDM and MCDM problems under conditions in which the weight information is completely unknown or known. In addition, the proposed method is fairly flexible to use; the parameter $\lambda$, which is involved in the generalized aggregation operators, can affect the final results. Therefore, Decision Makers (DMs) can choose appropriate $\lambda$ according to their preferences to obtain the most satisfactory result.

Although our proposed method can efficiently address the MCGDM/MCDM problems involving a large amount of data using aggregation operators, it allows the evaluations under different criteria to compensate each other in the aggregation process. In some problems, the criteria evaluations cannot compensate each other and the non-compensation among different criteria should be considered sufficiently. To solve such kinds of problems, future research will focus on incorporating the non-compensation principle into fuzzy aggregation operators to develop feasible MCGDM/MCDM methods.

\section{Acknowledgements}

The authors are very grateful to the anonymous reviewers for their valuable comments and suggestions to help improve the overall quality of this paper.
This work was supported by the National Natural Science Foundation of China (No. 71901226) and Hunan Provincial Natural Science Foundation of China (Nos. 2019JJ51001 and 2018JJ3131).

\section{References}

1. Kim, S.H. and Ahn, B.S. "Interactive group decision making procedure under incomplete information", European Journal of Operational Research, 116(3), pp. 498-507 (1999).

2. Garg, H. and Kaur, G. "Extended TOPSIS method for multi-criteria group decision-making problems under cubic intuitionistic fuzzy environment", Scientia Iranica, 27(1), pp. 396-410 (2020). https://doi.org/10.24200/sci.2018.5307.1194

3. Zadeh, L.A. "Fuzzy sets", Information and Control, 8(3), pp. 338-353 (1965).

4. Wang, Y., Duan, X., Liu, X., Wang, C., and Li, Z. "A spectral clustering method with semantic interpretation based on axiomatic fuzzy set theory", Applied Soft Computing, 64, pp. 59-74 (2018).

5. Sonmez, O. and Bizimana, H. "Flood hazard risk evaluation using fuzzy logic and weightage based combination methods in Geographic Information System (GIS)", Scientia Iranica, 27(2), pp. 517-528 (2020). https://doi.org/10.24200/SCI.2018.21037

6. Atanassov, K.T. "Intuitionistic fuzzy sets", Fuzzy Sets and Systems, 20, pp. 87-96 (1986).

7. Peng, H.G. and Wang, J.Q. "Cloud decision model for selecting sustainable energy crop based on linguistic intuitionistic information", International Journal of Systems Science, 48(15), pp. 3316-3333 (2017).

8. Tooranloo, H.S. and Sadat Ayatollah, A. "A model for failure mode and effects analysis based on intuitionistic fuzzy approach", Applied Soft Computing, 49, pp. 238247 (2016).

9. Zhou, X., Shi, Y., Deng, X., and Deng, Y. "DDEMATEL: A new method to identify critical success factors in emergency management", Safety Science, 91, pp. 93-104 (2017).

10. Yazdi, M. "Risk assessment based on novel intuitionistic fuzzy-hybrid-modified TOPSIS approach", Safety Science, 110, pp. 438-448 (2018).

11. Shu, M.H., Cheng, C.H., and Chang, J.R. "Using intuitionistic fuzzy sets for fault tree analysis on printed circuit board assembly", Microelectronics Reliability, 46(12), pp. 2139-2148 (2006).

12. Zhang, X. and Liu, P. "Method for aggregating triangular intuitionistic fuzzy information and its application to decision-making", Technological and Economic Development of Economy, 16(2), pp. 280-290 (2010). 
13. Li, D.F. "A ratio ranking method of triangular intuitionistic fuzzy numbers and its application to MADM problems", Computers and Mathematics with Applications, 60(6), pp. 1557-1570 (2010).

14. Wan, S.P., Wang, Q.Y., and Dong, J.Y. "The extended VIKOR method for multi-attribute group decision making with triangular intuitionistic fuzzy numbers", Knowledge-Based Systems, 52, pp. 65-77 (2013).

15. Wang, J.Q. and Zhong, Z. "Aggregation operators on intuitionistic trapezoidal fuzzy number and its application to multi-criteria decision making problems", Journal of Systems Engineering and Electronics, 20(2), pp. 321-326 (2009).

16. Wan, S.P. and Yi, Z.H. "Power average of trapezoidal intuitionistic fuzzy numbers using strict t-norms and t-conorms", IEEE Transactions on Fuzzy Systems, 24(5), pp. 1035-1047 (2015).

17. Li, X. and Chen, X. "Value determination method based on multiple reference points under a trapezoidal intuitionistic fuzzy environment", Applied Soft Computing, 63, pp. 39-49 (2018).

18. Govindan, K. and Jepsen, M.B. "Supplier risk assessment based on trapezoidal intuitionistic fuzzy numbers and ELECTRE TRI-C: a case illustration involving service suppliers", Journal of the Operational Research Society, 67(2), pp. 339-376 (2016).

19. Yang, M.S. and Ko, C.H. "On a class of fuzzy cnumbers clustering procedures for fuzzy data", Fuzzy Sets and Systems, 84(1), pp. 49-60 (1996).

20. Li, D.Y., Liu, C.Y., and Liu, L.Y. "Study on the universality of the normal cloud model", Engineering Science, 6(8), pp. 28-34 (2004).

21. Wang, J.Q., Li, K.J., and Zhang, H.Y. "Multicriteria decision-making method based on induced intuitionistic normal fuzzy related aggregation operators", International Journal of Uncertainty, Fuzziness and Knowledge-Based Systems, 20(04), pp. 559-578 (2012).

22. Wang, J.Q., Zhou, P., Li, K.J., Zhang, H.Y., and Chen, X.H. "Multi-criteria decision-making method based on normal intuitionistic fuzzy-induced generalized aggregation operator", TOP, 22(3), pp. 1103-1122 (2014).

23. Liu, P. and Teng, F. "Multiple criteria decision making method based on normal interval-valued intuitionistic fuzzy generalized aggregation operator", Complexity, 21(5), pp. 277-290 (2016).

24. Harsanyi, J.C. "Cardinal welfare, "Individualistic ethics, and interpersonal comparisons of utility", Journal of Political Economy, 63(4), pp. 309-321 (1955).

25. Tian, C., Peng, J.J., Zhang, S., Zhang, W.Y., and Wang, J.Q. "Weighted picture fuzzy aggregation oper- ators and their applications to multi-criteria decisionmaking problems", Computers and Industrial Engineering, 137, p. 106037 (2019).

26. Xu, Z.S. and Da, Q.L. "The ordered weighted geometric averaging operators", International Journal of Intelligent Systems, 17(7), pp. 709-716 (2002).

27. Liu, F., Zhang, W.G., and Zhang, L.H. "A note on 'A group decision making model based on a generalized ordered weighted geometric average operator with interval preference matrices' ", Fuzzy Sets and Systems, 246, pp. 1-18 (2014).

28. Yager, R.R. "On ordered weighted averaging aggregation operators in multicriteria decision making", IEEE Transactions on Systems, Man, and Cybernetics, 18(1), pp. 183-190 (1988).

29. Wang, T., Liu, J., Li, J., Xue, Y., and Dai, H. "An intuitionistic fuzzy OWA-TOPSIS method for collaborative network formation considering matching characteristics", Scientia Iranica, 25(3), pp. 16711687 (2018).

30. Wang, J., Wang, J.Q., Tian, Z.P., and Zhao, D.Y. "A multi-hesitant fuzzy linguistic multi-criteria decisionmaking approach for logistics outsourcing with incomplete weight information", International Transactions in Operational Research, 25(3), pp. 831-856 (2018).

31. Jana, C., Pal, M., and Wang, J.Q. "Bipolar fuzzy Dombi prioritized weighted aggregation operators in multiple attribute decision making", Soft Computing, 24, pp. 3631-3646 (2020).

https://doi.org/10.1007/s00500-019-04130-z

32. Xu, Z.S. and Da, Q.L. "Hybrid aggregation operator and its application to multiple attribute decision making problems", Journal of Southeast University (English Edition), 19(2), pp. 174-177 (2003).

33. Liu, H.C., Liu, L., and Li, P. "Failure mode and effects analysis using intuitionistic fuzzy hybrid weighted Euclidean distance operator", International Journal of Systems Science, 45(10), pp. 2012-2030 (2014).

34. Tian, Z.P., Wang, J., Wang, J.Q., and Chen, X.H. "Multi-criteria decision-making approach based on gray linguistic weighted Bonferroni mean operator", International Transactions in Operational Research, 25(5), pp. 1635-1658 (2018).

35. Yager, R.R. "Generalized OWA aggregation operators", Fuzzy Optimization and Decision Making, 3(1), pp. 93-107 (2004).

36. Peng, H.G., Zhang, H.Y., Wang, J.Q., and Li, L. "An uncertain Z-number multicriteria group decisionmaking method with cloud models", Information Sciences, 501, pp. 136-154 (2019).

37. Chen, T., He, S.S., Wang, J.Q., Li, L., and Luo, H. "Novel operations for linguistic neutrosophic sets 
on the basis of Archimedean copulas and co-copulas and their application in multi-criteria decision-making problems", Journal of Intelligent and Fuzzy Systems, 37(2), pp. 2887-2912 (2019).

38. Xu, Z. "Intuitionistic fuzzy aggregation operators", IEEE Transactions on Fuzzy Systems, 15(6), pp. 11791187 (2007).

39. Xu, Z. and Yager, R.R. "Some geometric aggregation operators based on intuitionistic fuzzy sets", International Journal of General Systems, 35(4), pp. 417-433 (2006).

40. Zhao, H., Xu, Z., Ni, M., and Liu, S. "Generalized aggregation operators for intuitionistic fuzzy sets", International Journal of Intelligent Systems, 25(1), pp. 1-30 (2010).

41. Zhang, X. and Liu, P. "Method for aggregating triangular fuzzy intuitionistic fuzzy information and its application to decision making", Technological and Economic Development of Economy, 16(2), pp. 280290 (2010).

42. Wang, J.Q., Nie, R., Zhang, H.Y., and Chen, X.H. "New operators on triangular intuitionistic fuzzy numbers and their applications in system fault analysis", Information Sciences, 251, pp. 79-95 (2013).

43. Yu, D. "Prioritized information fusion method for triangular intuitionistic fuzzy set and its application to teaching quality evaluation", International Journal of Intelligent Systems, 28(5), pp. 411-435 (2013).

44. Wei, G. "Some arithmetic aggregation operators with intuitionistic trapezoidal fuzzy numbers and their application to group decision making", Journal of Computers, 5(3), pp. 345-351 (2010).

45. Wu, J. and Cao, Q.W. "Same families of geometric aggregation operators with intuitionistic trapezoidal fuzzy numbers", Applied Mathematical Modelling, 37(1-2), pp. 318-327 (2013).

46. Yager, R.R. "The power average operator", IEEE Transactions on Systems, Man, and Cybernetics-Part A: Systems and Humans, 31(6), pp. 724-731 (2001).

47. Xu, Z. and Yager, R.R. "Power-geometric operators and their use in group decision making", IEEE Transactions on Fuzzy Systems, 18(1), pp. 94-105 (2009).

48. Xu, Z. "Approaches to multiple attribute group decision making based on intuitionistic fuzzy power aggregation operators", Knowledge-Based Systems, 24(6), pp. 749-760 (2011).

49. Li, Y.Y., Wang, J.Q., and Wang, T.L. "A linguistic neutrosophic multi-criteria group decision-making approach with EDAS method", Arabian Journal for Science and Engineering, 44(3), pp. 2737-2749 (2019).
50. Xu, Z. "Fuzzy harmonic mean operators", International Journal of Intelligent Systems, 24(2), pp. 152172 (2009).

51. Liu, J., Chen, H., Zhou, L., Tao, Z., and He, Y. "On the properties of the generalized OWHA operators and their application to group decision making", Journal of Intelligent and Fuzzy Systems, 27(4), pp. 2077-2089 (2014).

52. Wei, G.W. "FIOWHM operator and its application to multiple attribute group decision making", Expert Systems with Applications, 38(4), pp. 2984-2989 (2011).

53. Liu, P., Zhang, X., and Jin, F. "A multi-attribute group decision-making method based on intervalvalued trapezoidal fuzzy numbers hybrid harmonic averaging operators", Journal of Intelligent and Fuzzy Systems, 23(5), pp. 159-168 (2012).

54. Peng, H.G., Shen, K.W., He, S.S., Zhang, H.Y., and Wang, J.Q. "Investment risk evaluation for new energy resources: An integrated decision support model based on regret theory and ELECTRE III", Energy Conversion and Management, 183, pp. 332-348 (2019).

55. Peng, H.G. and Wang, J.Q. "A multicriteria group decision-making method based on the normal cloud model with Zadeh's Z-numbers", IEEE Transactions on Fuzzy Systems, 26(6), pp. 3246-3260 (2018).

56. Peng, H.G., Zhang, H.Y., and Wang, J.Q. "Cloud decision support model for selecting hotels on TripAdvisor. com with probabilistic linguistic information", International Journal of Hospitality Management, 68, pp. 124-138 (2018).

57. Kumar, T., Bajaj, R., and Ansari, M.D. "On accuracy function and distance measures of intervalvalued Pythagorean fuzzy sets with application in decision making", Scientia Iranica, 27(4), pp. 21272139 (2020). https://doi.org/10.24200/SCI.2019.51579.2260

58. Wang, J.Q. and Li, K.J. "Multi-criteria decisionmaking method based on intuitionistic normal fuzzy aggregation operators", Systems Engineering Theory and Practice, 33(6), pp. 1501-1508 (2013).

59. Szmidt, E. and Kacprzyk, J. "Ranking alternatives expressed via Atanassov's intuitionistic fuzzy sets", Proceedings of IPMU, 8, pp. 1604-1611 (2008).

60. Peng, H.G., Wang, J.Q., and Cheng, P.F. "A linguistic intuitionistic multi-criteria decision-making method based on the Frank Heronian mean operator and its application in evaluating coal mine safety", International Journal of Machine Learning and Cybernetics, 9(6), pp. 1053-1068 (2018).

\section{Biographies}

Hong-Gang Peng is a PhD degree candidate in Management Science and Engineering at the Business School of Central South University. His research interests include information management, and decisionmaking theory, and applications. 
Jing Wang is an Associate Professor in the College of Logistics and Transportation, Central South University of Forestry and Technology, China. She received her $\mathrm{PhD}$ degree in Management Science and Engineering from the School of Business, Central South University in 2016. She also holds an MSc degree in Information Engineering from University of Osnabrueck, Germany in 2006. Her current research focuses on decision-making theory and applications as well as quality management.
Jian-Qiang Wang is a Professor at the Department of Management Science and Information Management at the Business School of Central South University. He holds a PhD in Management Science \& Engineering and he is also a PhD Supervisor in this major. Over the past couple decades, his research interests are in the area of decision-making theory. His current research interests include information management, and decision-making theory and applications, and risk management and control. 